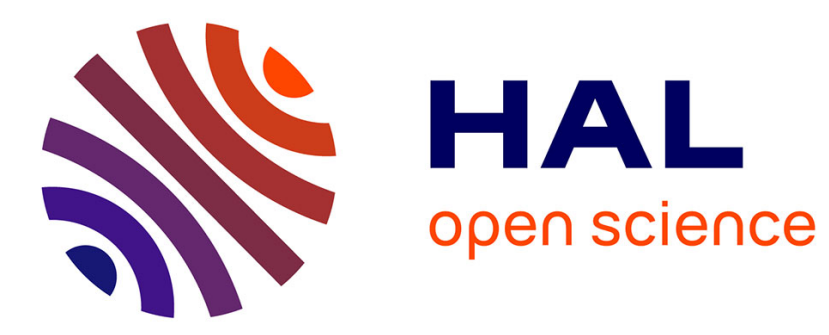

\title{
Energy Decay and Stability of a Perfectly Matched Layer For the Wave Equation
}

\author{
Daniel Henri Baffet, Marcus J. Grote, Sébastien Imperiale, Maryna \\ Kachanovska
}

\section{- To cite this version:}

Daniel Henri Baffet, Marcus J. Grote, Sébastien Imperiale, Maryna Kachanovska. Energy Decay and Stability of a Perfectly Matched Layer For the Wave Equation. Journal of Scientific Computing, 2019, 10.1007/s10915-019-01089-9 . hal-01865484v2

\section{HAL Id: hal-01865484 \\ https://hal.science/hal-01865484v2}

Submitted on 15 Nov 2019

HAL is a multi-disciplinary open access archive for the deposit and dissemination of scientific research documents, whether they are published or not. The documents may come from teaching and research institutions in France or abroad, or from public or private research centers.
L'archive ouverte pluridisciplinaire HAL, est destinée au dépôt et à la diffusion de documents scientifiques de niveau recherche, publiés ou non, émanant des établissements d'enseignement et de recherche français ou étrangers, des laboratoires publics ou privés. 


\title{
Energy Decay and Stability of a Perfectly Matched Layer For the Wave Equation
}

\author{
Daniel H. Baffet • Marcus J. Grote . \\ Sébastien Imperiale • Maryna Kachanovska
}

November 15, 2019

\begin{abstract}
In [26,25], a PML formulation was proposed for the wave equation in its standard second-order form. Here, energy decay and $L^{2}$ stability bounds in two and three space dimensions are rigorously proved both for continuous and discrete formulations with constant damping coefficients. Numerical results validate the theory.
\end{abstract}

\section{Introduction}

In the last two decades, the perfectly matched layer (PML) approach [13] has proved a flexible and accurate method for the simulation of waves in unbounded media. It consists in surrounding the region of interest by an absorbing layer, which generates no reflections at its interface; hence, it is perfectly matched. As the waves propagate through the layer, they decay exponentially until becoming vanishingly small at the outer boundary of the computational domain, where any stable boundary condition can be imposed. Due to its simplicity, versatility and robust treatment of corners, Bérenger's perfectly matched layer (PML) approach

D. Baffet

Department of Mathematics and Computer Science, University of Basel, Spiegelgasse 1, 4051 Basel, Switzerland

E-mail: daniel.baffet@unibas.ch

M. J. Grote

Department of Mathematics and Computer Science, University of Basel, Spiegelgasse 1, 4051 Basel, Switzerland

E-mail: marcus.grote@unibas.ch

S. Imperiale

Inria - LMS, Ecole Polytechnique, CNRS - Institut Polytechnique de Paris, Palaiseau, France

E-mail: sebastien.imperiale@inria.fr

M. Kachanovska

POEMS (UMR 7231 CNRS, ENSTA, INRIA), INRIA, Institut Polytechnique de Paris, Palaiseau, France

E-mail: maryna.kachanovska@inria.fr 
[13] for Maxwell's equations quickly gained in popularity and was soon extended to other first-order hyperbolic equations $[28,2,19]$.

The original PML formulation $[13,14]$ was based on splitting the electromagnetic fields into two parts, the first containing the tangential derivatives and the second containing the normal derivatives; damping was then enforced only upon the normal component. Abarbanel and Gottlieb [1] showed that Bérenger's "splitfield" approach was only weakly well-posed. Several strongly well-posed "unsplit" formulations were then proposed, some of which were shown to be linearly equivalent $[3,36]$. Well-posedness, however, does not prevent exponential growth of the solution while even the stronger notion of stability generally allows for polynomial growth in time. In fact, both split and unsplit PML formulations can generate late-time linear growth $[1,7]$, an undesirable behavior which was later removed through an alternative complex frequency shifted (CFS) scaling function [12].

Although stable PML formulations existed for a variety of wave equations, exponential growth was observed in various models involving anisotropy. In [6], Bécache, Fauqueux and Joly derived a necessary condition for the stability of PML for general hyperbolic systems based on the geometrical properties of the dispersion relation. Related to the existence of backward propagating waves, this condition explains in particular instabilities observed in anisotropic elasticity and led to necessary and sufficient stability conditions for orthotropic elastic waves. Appelö, Hagstrom and Kreiss [3] also derived necessary and sufficient stability conditions for first order constant coefficient Cauchy problems; they require verifying a number of algebraic inequalities in Fourier-Laplace domain, but also yield an energy in physical space that involves combinations of higher order derivatives of the unknowns and decays with time - see also [27]. In recent years, stable PML formulations were proposed for linearized Euler equations [29,34], anisotropic acoustics [20], aeroacoustics [21], short water waves [5] and electromagnetic dispersive media $[9,10,11,8]$.

Even when the geometric stability condition [6] guarantees the temporal stability of the Cauchy problem, physical boundaries and interfaces that interact with the PML can induce new instabilities. However, if the complex change of variables in the Laplace domain is applied not only to the normal derivatives inside the PML but also to the tangential derivatives at the physical boundary condition, the initial-boundary value problem generally inherits the stability of the Cauchy problem [23].

By using the Cagniard-De Hoop technique, Diaz and Joly [21] proved the exponential accuracy of PMLs with respect to the damping coefficient and the layer's thickness. Convergence for two-dimensional scattering problems with an annular PML was proved in [16]. Exponential decay of the energy both for a continuous and a semi-discrete PML formulation for the one-dimensional wave equation was proved in [24]. In two or more space dimensions, however, the derivation of stability estimates for the transmission problem associated with a PML using energy techniques, well suited for any subsequent numerical analysis, still remains an open question.

In the frequency domain, PML formulations essentially consist of a complexvalued coordinate stretching across the damping layer [17]. The inverse Fourier transformation back to the time domain, however, is more intricate and generally introduces additional unknowns. Moreover, initial PML formulations for time-dependent wave equations in second-order form required first reformulat- 
ing them as first-order hyperbolic systems, thereby introducing many additional unknowns. In $[33,35,4]$, various PML formulations were derived for second-order wave equations from acoustics, electromagnetics and elasticity. Still, the inverse Fourier transformation of the PML system in the frequency domain typically led to convolution integrals in the time domain [33].

In $[26,25]$, Grote and Sim proposed an efficient PML formulation directly for the wave equation in its second-order form, which avoids convolution integrals while keeping minimal the number of auxiliary variables; in fact, it requires only two auxiliary variables in two dimensions and four auxiliary variables in three dimensions inside the absorbing layer. As it avoids convolution integrals, it is also local in time and easily coupled with standard finite difference or finite element methods. Moreover, discontinuous absorption coefficients can be used without special treatment of the transmission conditions (i.e. without introducing boundary terms) as they are naturally taken into account into the variational formulation, unlike in e.g. [20]. Kaltenbacher, Kaltenbacher and Sim [32] addressed the stability of the PML formulation from $[26,25]$ via an energy analysis and also applied it to aeroacoustics. By "omitting one critical term involving the mixed products of the damping functions", they were able to prove long-time stability of a reduced (rPML) formulation which, however, "will not achieve perfect matching" [32].

Here, we consider the original PML formulation from $[26,25]$ for the wave equation in its standard second-order form. In Section 2, as a first step towards analyzing more general higher-dimensional transmission problems, we prove energy decay both in two and three space dimensions for the PML system of $[26,25]$ with constant damping functions. The key distinguishing features of our analysis is that it avoids Laplace/Fourier transforming the problem into the frequency domain and thus explicitly yields a new (space-time) energy of the PML system including finite thickness and corners. These results then imply boundedness of all the unknowns in the $L^{2}$-norm. Next, in Section 3, we derive similar estimates for the semi-discrete and the fully discrete case. Finally, in Section 4, we present numerical results which validate the theory.

\section{Continuous formulation and energy estimates}

We consider the wave equation in its standard second-order form with constant unit wave speed inside a three dimensional rectangular region of interest, $\Omega_{0}$. To avoid spurious reflections from the boundary of $\Omega_{0}$, we surround it by a perfectly matched layer (PML), $\Omega_{p m l}$, truncated by a rectangular outer boundary, $B$. Inside the computational domain $\Omega$, the interior of $\overline{\Omega_{0} \cup \Omega_{p m l}}$, we consider the PML formulation of $[25,26]$ :

$$
\left\{\begin{array}{l}
\partial_{t}^{2} u+\operatorname{tr} \Gamma_{1} \partial_{t} u+\operatorname{tr} \Gamma_{3} u+\operatorname{det} \Gamma_{1} \psi-\Delta u-\operatorname{div} \phi=0 \\
\partial_{t} \psi=u \\
\partial_{t} \phi+\Gamma_{1} \phi=\Gamma_{2} \nabla u+\Gamma_{3} \nabla \psi
\end{array}\right.
$$


where, $u, \psi:(0, T) \times \Omega \rightarrow \mathbb{R}$ and $\phi:(0, T) \times \Omega \rightarrow \mathbb{R}^{3}$. The matrices $\Gamma_{1}, \Gamma_{2}$ and $\Gamma_{3}$ are defined as

$$
\Gamma_{1}=\left(\begin{array}{ccc}
\xi_{1} & 0 & 0 \\
0 & \xi_{2} & 0 \\
0 & 0 & \xi_{3}
\end{array}\right), \quad \Gamma_{2}=\left(\begin{array}{ccc}
\xi_{2}+\xi_{3}-\xi_{1} & 0 & 0 \\
0 & \xi_{1}+\xi_{3}-\xi_{2} & 0 \\
0 & 0 & \xi_{1}+\xi_{2}-\xi_{3}
\end{array}\right)
$$

and

$$
\Gamma_{3}=\left(\begin{array}{ccc}
\xi_{2} \xi_{3} & 0 & 0 \\
0 & \xi_{1} \xi_{3} & 0 \\
0 & 0 & \xi_{2} \xi_{1}
\end{array}\right)
$$

where each damping function $\xi_{i}$ only depends on the $i$-th spatial coordinate $x_{i}$, is non-negative throughout $\Omega$ and identically zero inside the (physical) region $\Omega_{0}$. Note that $\Gamma_{1}$ is defined here with the opposite sign with respect to the original formulation in $[25,26]$.

The PML system (2.1) must be completed with appropriate initial conditions in $\Omega$ and boundary conditions on $B$. Inside $\Omega_{p m l}$, all the initial conditions (and source terms) are identically zero. On the outer boundary $B$, we impose either homogeneous Dirichlet or Neumann conditions,

Dirichlet: $u=0 \quad$ (D), Neumann: $\partial_{\nu} u+\phi \cdot \boldsymbol{\nu}=0 \quad(\mathrm{~N})$.

We do not consider here Robin or absorbing boundary conditions (see for instance [18] or [22]), however our analysis could be extended to include more general boundary conditions.

When the PML is used only in a single direction, i.e. when $\xi_{i} \not \equiv 0$ and $\xi_{j}=$ $\xi_{k} \equiv 0$, for $i \neq j \neq k$, both $\Gamma_{3}$ and $\operatorname{det} \Gamma_{1}$ are zero and equations (2.1a) and (2.1c) no longer involve $\psi$; then, the above PML formulation involves only the three (scalar) auxiliary variables $\phi_{i}, i=1,2,3$. At a corner, however, where $\xi_{i} \xi_{j} \not \equiv 0$ for some $i \neq j$, the above formulation requires the four auxiliary variables $\psi$ and $\phi_{i}$, $i=1,2,3$.

To derive energy estimates for (2.1), we assume that the damping functions $\xi_{1}, \xi_{2}, \xi_{3}$ are constant throughout $\Omega$. We note that to the best of our knowledge there is no energy-based proof of the stability of the PML system with non-constant coefficients (and it seems that this question is highly non-trivial). The stability analysis of the PML system with constant coefficients is justified by the fact that when the damping profiles are piecewise constant, the PML system (2.1) can be written as a transmission problem between (2.1) with $\xi_{1}=\xi_{2}=\xi_{3}=0$ stated in $\Omega_{0}$ and (2.1) with piecewise-constant $\xi_{1}, \xi_{2}, \xi_{3}$ inside $\Omega_{p m l}$, each of which can also be split into multiple transmission problems with constant $\xi_{1}, \xi_{2}, \xi_{3}$ and appropriate transmission conditions - see [20]. Thus to analyze the stability of such a transmission problem, it is natural to first analyze the stability of each of the corresponding boundary value problems.

We introduce the following notations. Given $\mathbf{u}, \mathbf{v}: \Omega \rightarrow \mathbb{R}^{n}, n \geq 1$, we let

$$
\langle\mathbf{u}, \mathbf{v}\rangle=\int_{\Omega} \sum_{k=1}^{n} u_{k}(\mathbf{x}) v_{k}(\mathbf{x}) d \mathbf{x}, \quad\|\mathbf{u}\|=\langle\mathbf{u}, \mathbf{u}\rangle^{\frac{1}{2}} .
$$

More generally, for any given $n \times n$ symmetric positive semidefinite matrix $M$, we let

$$
\langle\mathbf{u}, \mathbf{v}\rangle_{M}=\int \mathbf{u}^{T}(\mathbf{x}) M \mathbf{v}(\mathbf{x}) d \mathbf{x}, \quad\|\mathbf{u}\|_{M}^{2}=\langle\mathbf{u}, \mathbf{u}\rangle_{M}
$$


when $M=m$ Id, with $m>0$ scalar, we write $\langle\cdot, \cdot\rangle_{m}$ instead of $\langle\cdot, \cdot\rangle_{m}$ Id . Moreover, given $\mathbf{u}, \mathbf{v}: \mathbb{R}_{+} \rightarrow\left(L^{2}(\Omega)\right)^{n}$, we will use the notation

$$
\langle\mathbf{u}, \mathbf{v}\rangle \equiv\langle\mathbf{u}, \mathbf{v}\rangle(t) \equiv\langle\mathbf{u}(t), \mathbf{v}(t)\rangle,
$$

and similarly for $\langle\cdot, \cdot\rangle_{M}$.

\subsection{Two-dimensional formulation}

In two space dimensions, the PML formulation (2.1) reduces to:

$$
\left\{\begin{array}{l}
\partial_{t}^{2} u+\operatorname{tr}\left(\Gamma_{1}\right) \partial_{t} u+\operatorname{det}\left(\Gamma_{1}\right) u-\Delta u-\operatorname{div} \phi=0 \\
\partial_{t} \phi+\Gamma_{1} \phi=\Gamma_{2} \nabla u
\end{array}\right.
$$

where the matrices $\Gamma_{1}$ and $\Gamma_{2}$ are given by

$$
\Gamma_{1}=\left(\begin{array}{cc}
\xi_{1} & 0 \\
0 & \xi_{2}
\end{array}\right), \quad \Gamma_{2}=\left(\begin{array}{cc}
\xi_{2}-\xi_{1} & 0 \\
0 & \xi_{1}-\xi_{2}
\end{array}\right) .
$$

Here, only two damping functions $\xi_{1}, \xi_{2}$ and two auxiliary variables $\phi_{1}, \phi_{2}$ are needed.

With the above 2D PML system we associate the following energy functional:

$$
E[u, \phi]=\frac{1}{2}\left(\left\|\partial_{t} u+a u\right\|^{2}+\|\nabla u+\phi\|^{2}+\|\phi\|_{a^{-1} \Gamma_{1}}^{2}+\|u\|_{b}^{2}\right),
$$

where

$$
a=\operatorname{tr} \Gamma_{1}, \quad b=\operatorname{det} \Gamma_{1} .
$$

In [7], a similar energy was shown to decay for a single PML layer formulation with positive constant damping coefficients for the 2D transverse electric (TE) Maxwell equations. Since the PML formulation (2.3) for the second-order wave equation differs from the first-order formulation in [7], we provide a proof of energy decay, which also paves the way to the analysis in the $3 \mathrm{D}$ case.

Theorem 2.1 Let $(u, \phi)$ be a sufficiently regular solution of (2.3) with constant damping coefficients $\xi_{1}, \xi_{2}$. Then

$$
\frac{d}{d t} E[u, \phi]=-\left(\|\nabla u+\phi\|_{\Gamma_{1}\left(\operatorname{Id}+a^{-1} \Gamma_{1}\right)}^{2}+\|\nabla u\|_{a^{-1} b}^{2}+\|u\|_{a b}^{2}\right) .
$$

Hence, $E[u, \phi]$ is a nonincreasing function of $t$.

Proof We write (2.3a) as

$$
\partial_{t}^{2} u+a \partial_{t} u+b u=\operatorname{div} \boldsymbol{\lambda}
$$

where $a, b$ are defined in (2.4) and $\boldsymbol{\lambda}=\boldsymbol{\nabla} u+\phi$. By adding $\boldsymbol{\nabla} \partial_{t} u+\Gamma_{1} \boldsymbol{\nabla} u$ to both sides of $(2.3 \mathrm{~b})$ we obtain

$$
\partial_{t} \boldsymbol{\lambda}+\Gamma_{1} \boldsymbol{\lambda}=\boldsymbol{\nabla} \partial_{t} u+\widetilde{\Gamma}_{2} \boldsymbol{\nabla} u
$$


where

$$
\widetilde{\Gamma}_{2}=\Gamma_{2}+\Gamma_{1}=\left(\begin{array}{cc}
\xi_{2} & 0 \\
0 & \xi_{1}
\end{array}\right)
$$

Testing (2.6a) with $\partial_{t} u+a u$ yields

$$
\frac{1}{2} \frac{d}{d t}\left(\left\|\partial_{t} u+a u\right\|^{2}+b\|u\|^{2}\right)+\|u\|_{a b}^{2}=-\left\langle\nabla\left(\partial_{t} u+a u\right), \boldsymbol{\lambda}\right\rangle .
$$

Next we test $(2.6 \mathrm{~b})$ with

$$
\boldsymbol{g}=\left(\operatorname{Id}+a^{-1} \Gamma_{1}\right) \boldsymbol{\lambda}-a^{-1} \Gamma_{1} \nabla u .
$$

The inner product of $\boldsymbol{g}$ with the left hand side of (2.6b) then yields

$$
\begin{aligned}
\left\langle\boldsymbol{g}, \partial_{t} \boldsymbol{\lambda}+\Gamma_{1} \boldsymbol{\lambda}\right\rangle= & \frac{1}{2} \frac{d}{d t}\left(\|\boldsymbol{\lambda}\|^{2}+\|\boldsymbol{\lambda}\|_{a^{-1} \Gamma_{1}}^{2}\right)+\|\boldsymbol{\lambda}\|_{\Gamma_{1}\left(\mathrm{Id}+a^{-1} \Gamma_{1}\right)}^{2} \\
& -\left\langle a^{-1} \Gamma_{1} \boldsymbol{\nabla} u, \partial_{t} \boldsymbol{\lambda}+\Gamma_{1} \boldsymbol{\lambda}\right\rangle .
\end{aligned}
$$

The inner product of $\boldsymbol{g}$ with the right hand side of (2.6b) leads to

$$
\begin{aligned}
\left\langle\boldsymbol{g}, \boldsymbol{\nabla} \partial_{t} u+\widetilde{\Gamma}_{2} \nabla u\right\rangle & =\left\langle\left(\operatorname{Id}+a^{-1} \Gamma_{1}\right) \boldsymbol{\lambda}, \boldsymbol{\nabla} \partial_{t} u+\widetilde{\Gamma}_{2} \nabla u\right\rangle \\
& -\frac{1}{2} \frac{d}{d t}\|\nabla u\|_{a^{-1} \Gamma_{1}}^{2}-\|\nabla u\|_{a^{-1} b}^{2}
\end{aligned}
$$

since $\Gamma_{1} \widetilde{\Gamma}_{2}=b$ Id.

Because of (2.6b), the right hand sides of the last two equations must be equal. By rearranging terms, we thus obtain

$$
\begin{gathered}
\frac{1}{2} \frac{d}{d t}\left(\|\boldsymbol{\lambda}\|^{2}+\|\boldsymbol{\lambda}\|_{a^{-1} \Gamma_{1}}^{2}+\|\boldsymbol{\nabla} u\|_{a^{-1} \Gamma_{1}}^{2}\right)+\|\boldsymbol{\lambda}\|_{\Gamma_{1}\left(\mathrm{Id}+a^{-1} \Gamma_{1}\right)}^{2}+\|\boldsymbol{\nabla} u\|_{a^{-1} b}^{2} \\
=\left\langle a^{-1} \Gamma_{1} \boldsymbol{\nabla} u, \partial_{t} \boldsymbol{\lambda}+\Gamma_{1} \boldsymbol{\lambda}\right\rangle+\left\langle\left(\operatorname{Id}+a^{-1} \Gamma_{1}\right) \boldsymbol{\lambda}, \boldsymbol{\nabla} \partial_{t} u+\widetilde{\Gamma}_{2} \boldsymbol{\nabla} u\right\rangle \\
=\frac{d}{d t}\langle\boldsymbol{\nabla} u, \boldsymbol{\lambda}\rangle_{a^{-1} \Gamma_{1}}+\left\langle\boldsymbol{\nabla} \partial_{t} u, \boldsymbol{\lambda}\right\rangle+\left\langle\left[a^{-1} \Gamma_{1}\left(\Gamma_{1}+\widetilde{\Gamma}_{2}\right)+\widetilde{\Gamma}_{2}\right] \boldsymbol{\nabla} u, \boldsymbol{\lambda}\right\rangle .
\end{gathered}
$$

It remains to deal with the terms on the right hand side of (2.8). First, we note that the term $\frac{d}{d t}\langle\nabla u, \boldsymbol{\lambda}\rangle_{a^{-1} \Gamma_{1}}$ can be expressed using the identity:

$$
\|\phi\|_{a^{-1} \Gamma_{1}}^{2}=\|\boldsymbol{\lambda}\|_{a^{-1} \Gamma_{1}}^{2}+\|\nabla u\|_{a^{-1} \Gamma_{1}}^{2}-2\langle\nabla u, \boldsymbol{\lambda}\rangle_{a^{-1} \Gamma_{1}} .
$$

Next, we simplify the last term of the right hand side of (2.8) by using

$$
a^{-1} \Gamma_{1}\left(\Gamma_{1}+\widetilde{\Gamma}_{2}\right)+\widetilde{\Gamma}_{2}=\Gamma_{1}+\widetilde{\Gamma}_{2}=a \mathrm{Id} .
$$

Substitution into (2.8) then yields

$$
\begin{gathered}
\frac{1}{2} \frac{d}{d t}\left(\|\boldsymbol{\lambda}\|^{2}+\|\boldsymbol{\phi}\|_{a^{-1} \Gamma_{1}}^{2}\right)+\|\boldsymbol{\lambda}\|_{\Gamma_{1}\left(\mathrm{Id}+a^{-1} \Gamma_{1}\right)}^{2}+\|\nabla u\|_{a^{-1} b}^{2} \\
\quad=\left\langle\boldsymbol{\nabla} \partial_{t} u+a \boldsymbol{\nabla} u, \boldsymbol{\lambda}\right\rangle
\end{gathered}
$$

Finally, we sum (2.7) and (2.9) to obtain (2.5), which concludes the proof. 


\subsection{Three-dimensional formulation}

We now return to the PML formulation (2.1) in its full three-dimensional setting. First, we prove a result on energy decay, analogous to Theorem 2.1 for the 2D case. To determine a judicious energy functional associated to (2.1), we study the coercivity of the sesquilinear form associated with our PML formulation in the Fourier-Laplace domain, following ideas from [31]; those calculations are not repeated here and we simply use the resulting expression for the energy. Then, we prove $L^{2}$ bounds on all the unknowns involved in (2.1).

\subsubsection{Energy Decay}

First, we introduce two additional unknowns, which are not present in (2.1) but are needed in our energy estimates below. For a solution $(u, \psi, \phi)$ of $(2.1)$, let

$$
\Psi(t)=\int_{0}^{t} \psi(\tau) d \tau, \quad \boldsymbol{\Phi}(t)=\int_{0}^{t} \boldsymbol{\phi}(\tau) d \tau+\boldsymbol{\Phi}(0),
$$

where $\boldsymbol{\Phi}(0)$ satisfies

$$
\Gamma_{1}\left(\Gamma_{1} \boldsymbol{\Phi}(0)+\phi(0)-\Gamma_{2} \nabla \psi(0)\right)=0 .
$$

This condition is required in the proof of Theorem 2.2 and imposes no true restriction. Indeed for sufficiently regular $\psi, \phi$, that is for $\Gamma_{1}\left(\phi(0)+\Gamma_{2} \nabla \psi(0)\right) \in$ $\left(L^{2}(\Omega)\right)^{3}$, we may always define $\boldsymbol{\Phi}(0)$ as follows: for each $i=1,2,3$, let $j$ and $k$ be such that $\{i, j, k\}=\{1,2,3\}$, and set $\Phi_{i}(0)=0$, if $\xi_{i}=0$, and

$$
\Phi_{i}(0)=-\xi_{i}^{-1} \phi_{i}(0)+\xi_{i}^{-1}\left(\xi_{j}+\xi_{k}-\xi_{i}\right) \partial_{x_{i}} \psi(0)
$$

otherwise. Next, we introduce the additional unknown $q$,

$$
q:=\partial_{t} u+\operatorname{tr} \Gamma_{1} u+\operatorname{tr} \Gamma_{3} \psi+\operatorname{det} \Gamma_{1} \Psi,
$$

which also plays an important role in the energy identity.

Using the above definitions, we associate with the 3D PML system (2.1) the energy functional

$$
E[u, \psi, \phi, \Phi, \Psi]=\frac{1}{2}\left(\|q\|^{2}+\|\nabla u+\phi\|^{2}+\left\|\Gamma_{1}(\nabla \psi+\boldsymbol{\Phi})\right\|^{2}\right) .
$$

The following theorem summarizes the principal result of this section.

Theorem 2.2 Let $(u, \psi, \phi)$ be a sufficiently regular solution of (2.1) with constant damping functions $\xi_{1}, \xi_{2}, \xi_{3}$. Then, the energy satisfies

$$
\frac{d}{d t} E[u, \psi, \phi, \Phi, \Psi]=-2\|\nabla u+\phi\|_{\Gamma_{1}}^{2},
$$

where $\boldsymbol{\Phi}$ and $\Psi$ are given by (2.10) and $\boldsymbol{\Phi}(0)$ satisfies (2.11). Hence, $E[u, \psi, \phi, \boldsymbol{\Phi}, \Psi]$ is nonincreasing in time.

For the proof we will need the following two lemmas. 
Lemma 2.1 Let $(u, \psi, \phi)$ be a sufficiently regular solution of (2.1) with constant damping functions, $\Psi$ and $\boldsymbol{\Phi}$ be defined by (2.10) with $\boldsymbol{\Phi ( 0 )}$ satisfying (2.11). Then q, defined in (2.12), satisfies

$$
\boldsymbol{\nabla} q=\partial_{t}^{2} \boldsymbol{\Lambda}+2 \Gamma_{1} \partial_{t} \boldsymbol{\Lambda}+\Gamma_{1}^{2} \boldsymbol{\Lambda},
$$

where

$$
\boldsymbol{\Lambda}=\boldsymbol{\nabla} \psi+\boldsymbol{\Phi}
$$

Proof Since $\xi_{i}=$ const, $i=1,2,3$, we can interchange $\nabla$ and multiplication by traces and determinants of the matrices $\Gamma_{1}, \Gamma_{3}$ in (2.12):

$$
\boldsymbol{\nabla} q=\partial_{t} \boldsymbol{\nabla} u+\operatorname{tr} \Gamma_{1} \boldsymbol{\nabla} u+\operatorname{tr} \Gamma_{3} \boldsymbol{\nabla} \psi+\operatorname{det} \Gamma_{1} \boldsymbol{\nabla} \Psi .
$$

We start by rewriting $\operatorname{tr} \Gamma_{1} \nabla u$ in (2.15) in a more convenient form. Since,

$$
\operatorname{tr} \Gamma_{1} \mathrm{Id}=\Gamma_{2}+2 \Gamma_{1}
$$

we have using (2.1c):

$$
\operatorname{tr} \Gamma_{1} \boldsymbol{\nabla} u=\Gamma_{2} \boldsymbol{\nabla} u+2 \Gamma_{1} \boldsymbol{\nabla} u=\partial_{t} \phi+\Gamma_{1} \phi-\Gamma_{3} \boldsymbol{\nabla} \psi+2 \Gamma_{1} \boldsymbol{\nabla} u .
$$

Inserting the above into (2.15) yields

$$
\begin{aligned}
\boldsymbol{\nabla} q & =\partial_{t}(\boldsymbol{\nabla} u+\phi)+\Gamma_{1} \phi-\Gamma_{3} \boldsymbol{\nabla} \psi+2 \Gamma_{1} \boldsymbol{\nabla} u+\operatorname{tr} \Gamma_{3} \boldsymbol{\nabla} \psi+\operatorname{det} \Gamma_{1} \boldsymbol{\nabla} \Psi \\
& =\partial_{t}(\boldsymbol{\nabla} u+\phi)+2 \Gamma_{1}(\boldsymbol{\nabla} u+\phi)-\Gamma_{1} \phi+\left(\operatorname{tr} \Gamma_{3} \mathrm{Id}-\Gamma_{3}\right) \boldsymbol{\nabla} \psi+\operatorname{det} \Gamma_{1} \boldsymbol{\nabla} \Psi
\end{aligned}
$$

Next, we use the two identities

$$
\operatorname{tr} \Gamma_{3} \mathrm{Id}-\Gamma_{3}=\Gamma_{1}\left(\Gamma_{1}+\Gamma_{2}\right), \quad \operatorname{det} \Gamma_{1} \mathrm{Id}=\Gamma_{1} \Gamma_{3},
$$

together with (2.14) to obtain

$$
\boldsymbol{\nabla} q=\partial_{t}^{2} \boldsymbol{\Lambda}+2 \Gamma_{1} \partial_{t} \boldsymbol{\Lambda}-\Gamma_{1} \phi+\Gamma_{1}\left(\Gamma_{1}+\Gamma_{2}\right) \boldsymbol{\nabla} \psi+\Gamma_{1} \Gamma_{3} \nabla \Psi
$$

or equivalently

$$
\boldsymbol{\nabla} q-\left(\partial_{t}^{2} \boldsymbol{\Lambda}+2 \Gamma_{1} \partial_{t} \boldsymbol{\Lambda}+\Gamma_{1}^{2} \boldsymbol{\Lambda}\right)=\Gamma_{1}\left(-\Gamma_{1} \boldsymbol{\Lambda}-\boldsymbol{\phi}+\left(\Gamma_{1}+\Gamma_{2}\right) \boldsymbol{\nabla} \psi+\Gamma_{3} \boldsymbol{\nabla} \Psi\right) .
$$

Thus, (2.13) holds true provided that

$$
\Gamma_{1}\left(-\Gamma_{1} \Phi-\phi+\Gamma_{2} \nabla \psi+\Gamma_{3} \nabla \Psi\right)=0
$$

If $\xi_{i}=0$, the $i$ th component of the vector on the left hand side automatically vanishes; otherwise, the result follows from Lemma 2.2, since $\boldsymbol{\Phi}(0)$ satisfies (2.11).

Remark 2.1 When $\xi_{i}, i=1,2,3$ are not constant and belong to $C^{1}(\Omega)$, it follows that

$$
\boldsymbol{\nabla} q=\partial_{t}^{2} \boldsymbol{\Lambda}+2 \Gamma_{1} \partial_{t} \boldsymbol{\Lambda}+\Gamma_{1}^{2} \boldsymbol{\Lambda}+u \boldsymbol{\nabla} \operatorname{tr} \Gamma_{1}+\psi \boldsymbol{\nabla} \operatorname{tr} \Gamma_{3}+\Psi \nabla \operatorname{det} \Gamma_{1}
$$


Lemma 2.2 Let $\Psi, \boldsymbol{\Phi}$ be defined by (2.10), with $\boldsymbol{\Phi ( 0 )}$ satisfying (2.11), and the indices $i, j, k \in\{1,2,3\}$ be all different. If $\xi_{i} \neq 0$, then

$$
\phi_{i}(t)+\xi_{i} \Phi_{i}(t)=\left(\xi_{j}+\xi_{k}-\xi_{i}\right) \partial_{x_{i}} \psi(t)+\xi_{j} \xi_{k} \partial_{x_{i}} \Psi(t), \quad t \geq 0 .
$$

Proof Let $\xi_{i} \neq 0$. Integrating the $i$-th component of (2.1c), we obtain using (2.10)

$$
\phi_{i}(t)-\phi_{i}(0)+\xi_{i}\left(\Phi_{i}(t)-\Phi_{i}(0)\right)=\left(\xi_{j}+\xi_{k}-\xi_{i}\right) \partial_{x_{i}}(\psi(t)-\psi(0))+\xi_{j} \xi_{k} \partial_{x_{i}} \Psi(t) .
$$

Since (2.11) implies that $\phi_{i}(0)+\xi_{i} \Phi_{i}(0)=\left(\xi_{j}+\xi_{k}-\xi_{i}\right) \partial_{x_{i}} \psi(0)$, the terms evaluated at $t=0$ cancel each other, which completes the proof.

Now we have all the necessary ingredients to prove Theorem 2.2.

Proof (Theorem 2.2) We test equation (2.1a) with $q$, defined in (2.12). Integration by parts then yields

$$
\frac{1}{2} \frac{d}{d t}\|q\|^{2}+\langle\nabla u+\phi, \nabla q\rangle=\int_{B}(\nabla u+\phi) \cdot \boldsymbol{\nu} q
$$

If the Dirichlet boundary condition (2.2)(D) is imposed, by (2.12) it follows that $q=0$ on $B$ and thus the right hand side of $(2.20)$ vanishes. If the Neumann boundary condition $(2.2)(\mathrm{N})$ is used, the same conclusion holds. Therefore, in either case we have

$$
\frac{1}{2} \frac{d}{d t}\|q\|^{2}+\langle\nabla u+\phi, \nabla q\rangle=0
$$

From (2.13) and (2.14), we thus obtain the statement of the theorem:

$$
\frac{1}{2} \frac{d}{d t}\|q\|^{2}+\left\langle\partial_{t} \boldsymbol{\Lambda}, \partial_{t}^{2} \boldsymbol{\Lambda}+2 \Gamma_{1} \partial_{t} \boldsymbol{\Lambda}+\Gamma_{1}^{2} \boldsymbol{\Lambda}\right\rangle=0,
$$

or equivalently

$$
\frac{d}{d t}\left(\frac{1}{2}\|q\|^{2}+\frac{1}{2}\left\|\partial_{t} \boldsymbol{\Lambda}\right\|^{2}+\frac{1}{2}\left\|\Gamma_{1} \boldsymbol{\Lambda}\right\|^{2}\right)=-2\left\|\partial_{t} \boldsymbol{\Lambda}\right\|_{\Gamma_{1}}^{2} .
$$

Remark 2.2 If $\xi_{1}=\xi_{2}=\xi_{3}=0$, Theorem 2.2 implies the following bound for some constant $C \geq 0$, which depends only on the initial data:

$$
\|\nabla u(t)\|^{2}+\left\|\partial_{t} u(t)\right\|^{2} \leq C, t \geq 0 .
$$

Indeed, in this particular case (2.1) reduces to

$$
\partial_{t}^{2} u-\Delta u-\operatorname{div} \phi=0, \quad \partial_{t} \phi=0,
$$

and the energy identity of Theorem 2.2 reduces to

$$
\frac{d}{d t}\left(\left\|\partial_{t} u\right\|^{2}+\|\nabla u+\phi\|^{2}\right)=0
$$

Since $\|\phi(t)\|=\|\phi(0)\|$ for all $t \geq 0$, we infer the bound in (2.23). 
In practice the damping parameters, $\xi_{1}, \xi_{2}, \xi_{3}$ are not constant. The following remarks address two more realistic cases where the damping parameters may vary.

Remark 2.3 In the case when $\xi_{i}, i=1,2,3$, are non-constant, but sufficiently regular (for instance, $C^{1}(\Omega)$ ), it follows from Remark 2.1 and $(2.20)$, that

$$
\frac{d}{d t} E[u, \psi, \boldsymbol{\phi}, \boldsymbol{\Phi}, \Psi]=-2\left\|\partial_{t} \boldsymbol{\Lambda}\right\|_{\Gamma_{1}}^{2}-\left\langle\partial_{t} \boldsymbol{\Lambda}, u \boldsymbol{\nabla} \operatorname{tr} \Gamma_{1}+\psi \boldsymbol{\nabla} \operatorname{tr} \Gamma_{3}+\Psi \boldsymbol{\nabla} \operatorname{det} \Gamma_{1}\right\rangle .
$$

Using Gronwall's inequality, one can derive an exponential bound on the energy (which yields well-posedness).

Remark 2.4 If $\xi_{i}$, with $i=1,2,3$, are piecewise constant, a similar analysis can be carried out. For simplicity, consider the case of a PML applied only in the direction of $x_{1}$. Suppose $\Omega_{0}=(-1,0)^{3}, \Omega_{P M L}=(0,1) \times(-1,0)^{2}, \xi_{2}=\xi_{3}=0$ and $\xi_{1}\left(x_{1}\right)=0$, for $x_{1}<0$, and $\xi_{1}>0$ constant, for $x_{1} \geq 0$. Let $\Sigma=\left\{x_{1}=0\right\} \times[-1,0]^{2}$ be the interface between the physical domain $\Omega_{0}$ and the PML domain $\Omega_{P M L}$. The respective normal is then $\mathbf{e}_{1}=(1,0,0)$. For this formulation we have the identity

$$
\frac{d}{d t} E[u, \psi, \boldsymbol{\phi}, \boldsymbol{\Phi}, \Psi]=-2\left\|\partial_{t} \boldsymbol{\Lambda}\right\|_{\Gamma_{1}}^{2}-\int_{\Sigma}\left(\partial_{t} \boldsymbol{\Lambda} \cdot \mathbf{e}_{1}\right) u\left[\xi_{1}\right]_{\Sigma},
$$

where $[f]_{\Sigma}$, given by

$$
[f]_{\Sigma}=\lim _{\varepsilon \rightarrow 0+}(f(\varepsilon)-f(-\varepsilon)),
$$

represents the jump of $f$ on $\Sigma$. Since the last term of (2.24) is, in general, not positive, we can not conclude energy decay from equation (2.24). We remark that to obtain (2.24) we implicitly use the transmission conditions

$$
[u]_{\Sigma}=0 \text { and }\left[(\boldsymbol{\nabla} u+\phi) \cdot \mathbf{e}_{1}\right]_{\Sigma}=\left[\partial_{t} \boldsymbol{\Lambda} \cdot \mathbf{e}_{1}\right]_{\Sigma}=0
$$

which are a consequence of the requirement that the layer in $\Omega_{P M L}$ perfectly matches the medium inside $\Omega_{0}$ (see [30] for the related discussion). Note also that since $\phi(0)=0$ in $\Omega$, there holds $\phi(t)=0$ in the physical domain $\Omega_{0}$, and therefore, in general, $\left[\nabla u \cdot \mathbf{e}_{1}\right]_{\Sigma} \neq 0$.

\subsubsection{Control of the unknowns}

Theorem 2.2 does not immediately imply $u, \psi$ and $\phi$ do not grow in time. We clarify the behaviour of the unknowns solving (2.1) in the following theorem.

Assumption 1 The initial data for (2.1) satisfies

$$
u(0), \psi(0) \in H^{1}(\Omega), \quad \partial_{t} u(0) \in L^{2}(\Omega), \quad \phi(0) \in\left(L^{2}(\Omega)\right)^{3} .
$$

Theorem 2.3 Let $(u, \phi, \psi)$ solve (2.1) with initial data satisfying Assumption 1 and $\xi_{1}, \xi_{2}, \xi_{3} \geq 0$ constant. Then there exists a non-negative constant $C$, which depends only on the initial data and $\xi_{j}, j=1,2,3$, such that

- if $\xi_{i} \neq 0$ for some $i \in\{1,2,3\}$, then

$$
\|u(t)\|_{H^{1}}+\left\|\partial_{t} u(t)\right\|+\|\phi(t)\| \leq C, \text { for all } t \geq 0
$$


- if, additionally, $\xi_{j} \neq 0$ for some $j \neq i, j \in\{1,2,3\}$, then

$$
\|\psi(t)\|_{H^{1}} \leq C \text { for all } t \geq 0
$$

From these estimates, it appears that we control the $L^{2}$-norms of all the unknowns inside the PML. Indeed, when $\xi_{i} \neq 0, \xi_{j} \neq 0$ for some $i \neq j$, this precisely corresponds to the statement of the theorem. Still, when only one $\xi_{i}$ is nonzero, that is for a PML in a single direction, the norm of $\psi$ in fact is not necessarily bounded. In that particular situation, however, (2.1a) and (2.1c) decouple from (2.1b) - see the discussion below (2.1) - and hence $\psi$ can be excluded from the PML formulation.

The proof of Theorem 2.3 relies on Lemmas 2.4, 2.5 below, whose proofs extensively use the Gronwall lemma stated below.

Lemma 2.3 Let $v \in C^{1}\left([0, \infty) ; L^{2}(\Omega)\right)$ and $w$ be defined by

$$
w=\partial_{t} v+\gamma v, \quad \gamma>0
$$

and assume that for some constant $C_{w} \geq 0,\|w(t)\| \leq C_{w}$ uniformly for all $t \geq 0$. Then,

$$
\|v(t)\| \leq\|v(0)\|+\gamma^{-1} C_{w}, \quad\left\|\partial_{t} v(t)\right\| \leq 2 C_{w}+\gamma\|v(0)\|, \quad \text { for all } t \geq 0
$$

The estimate of Theorem 2.2 controls the norm of $q$ defined in (2.12). The following lemma shows that whenever $\xi_{i}>0$ for some $i \in\{1,2,3\}$, controlling $\|q(t)\|$ amounts to controlling $\|u(t)\|$.

Lemma 2.4 Let $(u, \psi, \phi)$ solve (2.1) with initial data satisfying Assumption 1 and $\xi_{1}, \xi_{2}, \xi_{3} \geq 0$ constant. Then there exists a constant $C>0$, which depends only on the initial data and $\xi_{j}, j=1,2,3$, such that

- if $\xi_{i} \neq 0$ for some $i \in\{1,2,3\}$, then for all $t \geq 0$,

$$
\|u(t)\| \leq C, \quad(2.27 \mathrm{a}) \quad\left\|\partial_{t} u(t)\right\| \leq C .
$$

- If, additionally, $\xi_{j} \neq 0, j \neq i$, then

$$
\|\psi(t)\| \leq C, \quad \text { for all } t \geq 0
$$

Proof In the following, we let $C$ denote a generic constant that depends only on the initial data and $\xi_{j}, j=1,2,3$. From Theorem 2.2 it follows that $q$, defined in (2.12), satisfies

$$
\|q(t)\| \leq C \quad \text { for all } t \geq 0
$$

We now consider the following three distinct cases:

1. $\xi_{i} \neq 0$ and $\xi_{j}=\xi_{k}=0, i \neq j \neq k, k \in\{1,2,3\}$. The bounds (2.27a, 2.27b) follow from $q \equiv \partial_{t} u+\xi_{i} u$ and a direct application of Lemma 2.3. 
2. $\xi_{i}, \xi_{j} \neq 0$ and $\xi_{k}=0$. Due to $\partial_{t} \psi=u$, we have

$$
q \equiv \partial_{t} u+\left(\xi_{i}+\xi_{j}\right) u+\xi_{i} \xi_{j} \psi=\left(\partial_{t}+\xi_{i}\right)\left(\partial_{t}+\xi_{j}\right) \psi
$$

By applying Lemma 2.3 with $v=\left(\partial_{t}+\xi_{j}\right) \psi$ and $\gamma=\xi_{i}$, we get (using $\partial_{t} \psi(0)=$ $u(0))$

$$
\begin{aligned}
\left\|\left(\partial_{t}+\xi_{j}\right) \psi(t)\right\| & \leq\left\|\partial_{t} \psi(0)+\xi_{j} \psi(0)\right\|+C \xi_{i}^{-1} \\
& \leq\|u(0)\|+\left\|\xi_{j} \psi(0)\right\|+C \xi_{i}^{-1}, \\
\left\|\partial_{t}\left(\partial_{t}+\xi_{j}\right) \psi(t)\right\| & \leq \xi_{i}\left(\|u(0)\|+\left\|\xi_{j} \psi(0)\right\|\right)+2 C .
\end{aligned}
$$

From Lemma 2.3 applied to the first expression above, we get (2.28). By applying Lemma 2.3 to the second expression and using $\partial_{t} \psi=u$, we deduce (2.27a) and $(2.27 \mathrm{~b})$.

3. $\xi_{1}, \xi_{2}, \xi_{3}>0$. In this case one can verify that

$$
q=\left(\partial_{t}+\xi_{1}\right)\left(\partial_{t}+\xi_{2}\right)\left(\partial_{t}+\xi_{3}\right) \Psi
$$

The desired bounds are obtained like in the previous case by multiple applications of Lemma 2.3. The bounds in these expressions depend only on the initial data for $\psi, u$, since $\Psi(0) \equiv 0$, and on $\xi_{j}, j=1,2,3$.

The next Lemma 2.5 shows that controlling $\|\nabla u+\phi\|$ and $\left\|\Gamma_{1}(\boldsymbol{\nabla} \psi+\boldsymbol{\Phi})\right\|$ allows to control $\phi$ and $\nabla u$.

Lemma 2.5 Let $(u, \phi, \psi)$ satisfy (2.1) with initial data satisfying Assumption 1 and $\xi_{1}, \xi_{2}, \xi_{3} \geq 0$ constant. Then there exists a constant $C>0$, which depends only on the initial data and $\xi_{i}, i=1,2,3$, such that

- if $\xi_{i} \neq 0$ for some $i=1,2,3$, then fora all $t \geq 0$,

$$
\|\nabla u(t)\| \leq C, \quad(2.30 \mathrm{a}) \quad\|\phi(t)\| \leq C .
$$

- If, additionally, $\xi_{j} \neq 0$, for some $j \in\{1,2,3\}, j \neq i$, then

$$
\|\nabla \psi(t)\| \leq C \text { for all } t \geq 0 .
$$

Proof In the following, let $C>0$ denote a generic non-negative constant, which depends only on $\xi_{j}, j=1,2,3$ and the initial data. Thanks to Theorem 2.2, we have

$$
\begin{aligned}
& \|\boldsymbol{\nabla} u(t)+\phi(t)\| \leq C, \\
& \left\|\Gamma_{1}(\boldsymbol{\nabla} \psi(t)+\boldsymbol{\Phi}(t))\right\| \leq C,
\end{aligned}
$$

uniformly for all $t \geq 0$. We now consider four separate cases.

1. $\xi_{i}=\xi_{j}=\xi_{k}=0$. Then (2.30a), (2.30b) follow directly from Theorem 2.2 , see Remark 2.2

2. $\xi_{i} \neq 0$, and $\xi_{j}=\xi_{k}=0$. Without loss of generality, let us assume that $i=1$. To prove (2.30a) and (2.30b), we proceed in two steps. 
- Uniform bounds on $\left\|\partial_{x_{1}} u(t)\right\|,\left\|\phi_{1}(t)\right\|$. From Lemma 2.2, we have

$$
\phi_{1}=-\xi_{1} \Phi_{1}-\xi_{1} \partial_{x_{1}} \psi
$$

Then, (2.33) immediately implies $\left\|\phi_{1}(t)\right\| \leq C$ for all $t \geq 0$, which together with (2.32), yields $\left\|\partial_{x_{1}} u(t)\right\| \leq C$ for all $t \geq 0$.

- Uniform bounds on $\left\|\partial_{x_{\ell}} u(t)\right\|,\left\|\phi_{\ell}(t)\right\|, \ell \neq 1$. Without loss of generality, we let $\ell=2$; the bound for $\ell=3$ can be shown similarly. Since $\xi_{2}=\xi_{3}=0$, the second component of $(2.1 \mathrm{c})$ reduces to

$$
\partial_{t} \phi_{2}=\xi_{1} \partial_{x_{2}} u
$$

Hence, $w:=\left(\partial_{t}+\xi_{1}\right) \xi_{1}^{-1} \phi_{2}=\partial_{x_{2}} u+\phi_{2}$ is controlled by (2.32). From Lemma 2.3, we thus conclude that

$$
\left\|\phi_{2}(t)\right\| \leq C, \quad \text { for all } t \geq 0,
$$

and, using (2.32), that a similar corresponding bound holds for $\partial_{x_{2}} u(t)$.

3. $\xi_{i} \neq 0, \xi_{j} \neq 0, \xi_{k} \equiv 0$. Without loss of generality, we let $i=1, j=2$ and $k=3$. To prove the three bounds $(2.30 \mathrm{a}),(2.30 \mathrm{~b})$, and $(2.31)$, we again proceed in two steps.

- Uniform bounds on $\left\|\partial_{x_{\ell}} u(t)\right\|,\left\|\partial_{x_{\ell}} \psi(t)\right\|,\left\|\phi_{\ell}(t)\right\|, \ell=1,2$. Without loss of generality, we let $\ell=1$; for $\ell=2$, the argument is essentially identical. By Lemma 2.2,

$$
\phi_{1}+\xi_{1} \Phi_{1}=\left(\xi_{2}-\xi_{1}\right) \partial_{x_{1}} \psi
$$

or, adding to both sides of the above identity $\partial_{x_{1}} u=\partial_{x_{1}} \partial_{t} \psi$,

$$
\phi_{1}+\partial_{x_{1}} u+\xi_{1} \Phi_{1}+\xi_{1} \partial_{x_{1}} \psi=\partial_{t} \partial_{x_{1}} \psi+\xi_{2} \partial_{x_{1}} \psi .
$$

The bounds (2.32) and (2.33) show that the $L^{2}$-norm of the left hand side of the above expression is uniformly bounded in $t \geq 0$. Applying Lemma 2.3 to $w=\partial_{t} \partial_{x_{1}} \psi+\xi_{2} \partial_{x_{1}} \psi$, we deduce that $\left\|\partial_{x_{1}} \psi(t)\right\|$ and $\left\|\partial_{x_{1}} \partial_{t} \psi(t)\right\|=\left\|\partial_{x_{1}} u(t)\right\|$ are uniformly bounded in time. From (2.32) a uniform bound on $\left\|\phi_{1}(t)\right\|$ immediately follows.

- Uniform bounds on $\left\|\partial_{x_{3}} u(t)\right\|,\left\|\partial_{x_{3}} \psi(t)\right\|,\left\|\phi_{3}(t)\right\|$. Note $(2.18)$ cannot be used here for $i=3$. Thus, to obtain a similar expression, we integrate from 0 to $t$ the third component of (2.1c). Using (2.10), we get

$$
\begin{aligned}
\phi_{3}(t)-\phi_{3}(0) & =\left(\xi_{1}+\xi_{2}\right) \partial_{x_{3}} \psi(t) \\
& -\left(\xi_{2}+\xi_{1}\right) \partial_{x_{3}} \psi(0)+\xi_{1} \xi_{2} \partial_{x_{3}} \Psi(t) .
\end{aligned}
$$

We now add $\partial_{x_{3}} u(t)$ to both sides of $(2.35)$ and use $\partial_{t} \psi=u$ to rewrite the resulting expression as

$$
\begin{aligned}
\phi_{3}(t)+\partial_{x_{3}} u(t)-\phi_{3}(0)+\left(\xi_{2}+\xi_{1}\right) \partial_{x_{3}} \psi(0) & =\left(\partial_{t}+\xi_{1}\right) \\
& \times\left(\partial_{t}+\xi_{2}\right) \partial_{x_{3}} \Psi(t) .
\end{aligned}
$$

The $L^{2}$-norm of the left hand side of the above is uniformly bounded in time thanks to (2.32). By applying Lemma 2.3 twice to the right-hand side, 
we obtain uniform bounds on $\left\|\partial_{x_{3}} u(t)\right\|$ and $\left\|\partial_{x_{3}} \psi(t)\right\|$. The bound on $\phi_{3}$ follows immediately from the triangle inequality applied to (2.32).

4. $\xi_{1}, \xi_{2}, \xi_{3} \neq 0$. To prove the three bounds (2.30a), (2.30b), and (2.31), we first derive uniform bounds on $\left\|\partial_{x_{1}} u(t)\right\|,\left\|\phi_{1}(t)\right\|,\left\|\partial_{x_{1}} \psi(t)\right\|$ (for the remaining components the bounds can be derived similarly). By adding $\partial_{x_{1}} u$ to both sides of (2.18) with $i=1$ and using (2.10), we obtain

$$
\phi_{1}+\partial_{x_{1}} u+\xi_{1}\left(\Phi_{1}+\partial_{x_{1}} \psi\right)=\left(\partial_{t}+\xi_{2}\right)\left(\partial_{t}+\xi_{3}\right) \partial_{x_{1}} \Psi .
$$

The left-hand side of the above equation is uniformly bounded due to (2.32) and (2.33). Then, we again apply twice Lemma 2.3 to the right-hand side, which allows us to bound the $L^{2}$-norms of $\partial_{x_{1}} \Psi, \partial_{x_{1}} \psi$ and $\partial_{x_{1}} u$ uniformly in time. Because of (2.32), we also control $\left\|\phi_{1}(t)\right\|$.

Proof (Theorem 2.3) The bounds of the theorem follow directly from Lemma 2.4 and Lemma 2.5 .

\section{Discretization of the PML system and energy estimates}

Here, we consider a discretization of the 3D PML system (2.1) and prove that it is stable by energy arguments similar to the analysis for the continuous case in Section 2. We start with a construction and analysis of an implicit scheme, because the derivation of the explicit discretization (and its stability analysis) will rely heavily on the respective results obtained for the implicit scheme. In fact, the explicit scheme can be seen as a slight modification of the implicit one, which allows us to prove that it retains the same stability properties under a standard CFL condition, independent of the damping functions inside the PML.

\subsection{Implicit scheme}

We consider an implicit semi-discretization in time of (2.1), based on the classical second-order $\theta$-scheme (see [15] for a complete convergence analysis) with $\theta=\frac{1}{4}$.

\subsubsection{Notation}

We denote by $v^{n} \approx v\left(t^{n}\right)$, where $t^{n}=n \Delta t$. Given a sequence $\left\{v^{n}\right\}_{n=0}^{\infty}$, we define for $n \geq 1$,

$$
\begin{aligned}
& {\left[v^{n}\right]_{\Delta t}=\frac{v^{n+1}-v^{n-1}}{2 \Delta t}, \quad\left[\left[v^{n}\right]\right]_{\Delta t}=\frac{v^{n+1}-2 v^{n}+v^{n-1}}{\Delta t^{2}},} \\
& \left\{v^{n}\right\}_{1 / 4}=\frac{v^{n+1}+2 v^{n}+v^{n-1}}{4}, \quad v^{n+1 / 2}=\frac{v^{n}+v^{n+1}}{2} .
\end{aligned}
$$

The following lemmas provide some useful algebraic identities. 
Lemma 3.1 Let two sequences $\left\{a^{n}\right\}_{n=0}^{\infty}$ and $\left\{b^{n}\right\}_{n=0}^{\infty}$ of elements in some vector space satisfy

$$
\frac{a^{n+1}-a^{n}}{\Delta t}=\frac{b^{n+1}+b^{n}}{2}, \quad n \geq 0
$$

Then, for each $n \geq 1$, the following identities hold:

$$
\left[a^{n}\right]_{\Delta t}=\frac{a^{n+1 / 2}-a^{n-1 / 2}}{\Delta t}=\left\{b^{n}\right\}_{1 / 4}, \quad\left[\left[a^{n}\right]\right]_{\Delta t}=\left[b^{n}\right]_{\Delta t} .
$$

The proof of Lemma 3.1 is straightforward and therefore omitted. In the sequel, we shall employ Lemma 3.1 repeatedly without making explicit reference to it.

The following algebraic result is classical and corresponds to the discrete counterpart of the continuous equalities: $v \partial_{t} v=\partial_{t} v^{2} / 2$ and $\partial_{t} v \partial_{t}^{2} v=\partial_{t}\left|\partial_{t} v\right|^{2} / 2$.

Lemma 3.2 For any sequence $\left\{v^{n}\right\}_{n=0}^{\infty}$, the following identities hold for all $n \geq 1$ :

$$
\begin{aligned}
& \left\{v^{n}\right\}_{1 / 4} \cdot\left[v^{n}\right]_{\Delta t}=\frac{1}{2 \Delta t}\left(\left|\frac{v^{n+1}+v^{n}}{2}\right|^{2}-\left|\frac{v^{n}+v^{n-1}}{2}\right|^{2}\right), \\
& {\left[v^{n}\right]_{\Delta t} \cdot\left[\left[v^{n}\right]\right]_{\Delta t}=\frac{1}{2 \Delta t}\left(\left|\frac{v^{n+1}-v^{n}}{\Delta t}\right|^{2}-\left|\frac{v^{n}-v^{n-1}}{\Delta t}\right|^{2}\right) .}
\end{aligned}
$$

Below we shall also use the following generalization of Lemma 3.2.

Lemma 3.3 For any sequences $\left\{v^{n}\right\}_{n=0}^{\infty},\left\{h^{n}\right\}_{n=0}^{\infty}$, the following identity holds for all $n \geq 1$ :

$$
\begin{aligned}
& \left(\left[v^{n}\right]_{\Delta t}+\left\{h^{n}\right\}_{1 / 4}\right) \cdot\left(\left[\left[v^{n}\right]\right]_{\Delta t}+\left[h^{n}\right]_{\Delta t}\right)=\frac{1}{2 \Delta t}\left(\left|r^{n+1 / 2}\right|^{2}-\left|r^{n-1 / 2}\right|^{2}\right), \\
& \text { where } \quad r^{\ell+\frac{1}{2}}=\frac{v^{\ell+1}-v^{\ell}}{\Delta t}+\frac{h^{\ell+1}+h^{\ell}}{2}, \quad \ell \geq 0 .
\end{aligned}
$$

The proofs of these two results are omitted here.

\subsubsection{Implicit semi-discretization}

We discretize (2.1a) with the implicit $\theta$-scheme as follows:

- discretize terms $v\left(t^{n}\right)$ by $\left\{v^{n}\right\}_{1 / 4}$,

- discretize terms $\partial_{t} v\left(t^{n}\right)$ by $\left[v^{n}\right]_{\Delta t}$,

- discretize terms $\partial_{t}^{2} v\left(t^{n}\right)$ by $\left[\left[v^{n}\right]\right]_{\Delta t}$.

Equations (2.1b) and (2.1c) are discretized using second-order finite differences centered about time $(n+1 / 2) \Delta t$. Then the semi-discrete version of (2.1) reads:

$$
\begin{aligned}
& {\left[\left[u^{n}\right]\right]_{\Delta t}+\operatorname{tr} \Gamma_{1}\left[u^{n}\right]_{\Delta t}+\operatorname{tr} \Gamma_{3}\left\{u^{n}\right\}_{1 / 4}+\operatorname{det} \Gamma_{1}\left\{\psi^{n}\right\}_{1 / 4}} \\
& -\Delta\left\{u^{n}\right\}_{1 / 4}-\operatorname{div}\left\{\phi^{n}\right\}_{1 / 4}=0, \\
& \frac{\psi^{n+1}-\psi^{n}}{\Delta t}=\frac{u^{n+1}+u^{n}}{2}, \\
& \frac{\phi^{n+1}-\phi^{n}}{\Delta t}+\Gamma_{1} \frac{\phi^{n+1}+\phi^{n}}{2}=\Gamma_{2} \nabla \frac{u^{n+1}+u^{n}}{2}+\Gamma_{3} \nabla \frac{\psi^{n+1}+\psi^{n}}{2},
\end{aligned}
$$


which is equipped with appropriate initial conditions for $\left(u^{0}, u^{1}, \psi^{0}, \phi^{0}\right)$. The last two equations imply

$$
\begin{aligned}
& {\left[\psi^{n}\right]_{\Delta t}=\left\{u^{n}\right\}_{1 / 4},} \\
& {\left[\phi^{n}\right]_{\Delta t}+\Gamma_{1}\left\{\phi^{n}\right\}_{1 / 4}=\Gamma_{2} \boldsymbol{\nabla}\left\{u^{n}\right\}_{1 / 4}+\Gamma_{3} \boldsymbol{\nabla}\left\{\psi^{n}\right\}_{1 / 4} .}
\end{aligned}
$$

Similarly to the setup of the continuous formulation, here we consider either of the following boundary conditions

Dirichlet: $u^{n}=0 \quad(\mathrm{D}), \quad$ Neumann: $\partial_{\nu} u^{n}+\boldsymbol{\phi}^{n} \cdot \boldsymbol{\nu}=0 \quad(\mathrm{~N})$,

on the boundary $B$ of $\Omega$.

Next, we introduce two auxiliary unknowns, $\boldsymbol{\Phi}^{n}$ and $\Psi^{n}$, in accordance with (2.10), as well as an auxiliary 'velocity' variable $v^{n}$ :

$$
\begin{aligned}
& \frac{\Psi^{n+1}-\Psi^{n}}{\Delta t}=\frac{\psi^{n+1}+\psi^{n}}{2}, \\
& \frac{\boldsymbol{\Phi}^{n+1}-\boldsymbol{\Phi}^{n}}{\Delta t}=\frac{\phi^{n+1}+\phi^{n}}{2} \\
& \frac{v^{n+1}+v^{n}}{2}=\frac{u^{n+1}-u^{n}}{\Delta t} .
\end{aligned}
$$

Again, we remark that (3.11) and (3.12) imply

$$
\begin{aligned}
& {\left[\Psi^{n}\right]_{\Delta t}=\left\{\psi^{n}\right\}_{1 / 4},} \\
& {\left[\boldsymbol{\Phi}^{n}\right]_{\Delta t}=\left\{\boldsymbol{\phi}^{n}\right\}_{1 / 4} .}
\end{aligned}
$$

For these equations to be consistent with (2.10), we also need to define initial conditions for $\Psi$ and $\boldsymbol{\Phi}$ - see (2.11) and the related discussion afterwards:

$$
\Psi^{0}=0, \quad \Gamma_{1}\left(\Gamma_{1} \boldsymbol{\Phi}^{0}+\phi^{0}-\Gamma_{2} \boldsymbol{\nabla} \psi^{0}\right)=0 .
$$

With this choice, the energy of the discrete system (3.7) corresponds to the energy of the continuous setting defined in Theorem 2.2. In particular, as previously,

$$
q^{n}:=v^{n}+\operatorname{tr} \Gamma_{1} u^{n}+\operatorname{tr} \Gamma_{3} \psi^{n}+\operatorname{det} \Gamma_{1} \Psi^{n}, \quad n \geq 0 .
$$

Using (3.1), we further introduce the notation:

$$
\begin{aligned}
& E_{k}^{n+\frac{1}{2}}=\frac{1}{2}\left\|q^{n+\frac{1}{2}}\right\|^{2} \\
& E_{p}^{n+\frac{1}{2}}=\frac{1}{2}\left(\left\|\nabla u^{n+\frac{1}{2}}+\phi^{n+\frac{1}{2}}\right\|^{2}+\left\|\Gamma_{1}\left(\nabla \psi^{n+\frac{1}{2}}+\boldsymbol{\Phi}^{n+\frac{1}{2}}\right)\right\|^{2}\right) \\
& E_{i m p l}^{n+\frac{1}{2}}=E_{k}^{n+\frac{1}{2}}+E_{p}^{n+\frac{1}{2}} .
\end{aligned}
$$

Here the subscript $k$ stands for 'kinetic', $p$ for 'potential' and $i m p l$ for 'implicit'.

With these notations, the energy decay result for the semi-discrete system (3.7) is summarized in the following theorem. 
Theorem 3.1 For any sufficiently regular solution $\left(u^{n}, \psi^{n}, \phi^{n}\right)$ of the initial-value problem for (3.7) it holds for all $n \geq 1$,

$$
\frac{1}{\Delta t}\left(E_{i m p l}^{n+\frac{1}{2}}-E_{i m p l}^{n-\frac{1}{2}}\right)=-2\left\|\left\{\nabla u^{n}+\phi^{n}\right\}_{1 / 4}\right\|_{\Gamma_{1}}^{2},
$$

where $E^{n+\frac{1}{2}}$ is defined in (3.20) and $\boldsymbol{\Phi}^{n}$ in (3.12) and satisfies (3.16).

The proof is based on the following two lemmas.

Lemma 3.4 For any sufficiently regular solution $\left(u^{n}, \psi^{n}, \phi^{n}\right)$ of the initial-value problem for (3.7), and $\boldsymbol{\Phi}^{n}, \Psi^{n}$ defined in $(3.11,3.12)$ and satisfying (3.16), it holds for $n \geq 1$ :

$$
\boldsymbol{\nabla}\left\{q^{n}\right\}_{1 / 4}=\left[\left[\boldsymbol{\Lambda}^{n}\right]\right]_{\Delta t}+2 \Gamma_{1}\left[\boldsymbol{\Lambda}^{n}\right]_{\Delta t}+\Gamma_{1}^{2}\left\{\boldsymbol{\Lambda}^{n}\right\}_{1 / 4},
$$

where

$$
\Lambda^{n}=\nabla \psi^{n}+\boldsymbol{\Phi}^{n}
$$

Proof The proof follows the derivation of Lemma 2.1. In particular, for $n \geq 1$,

$$
\boldsymbol{\nabla}\left\{q^{n}\right\}_{1 / 4}=\boldsymbol{\nabla}\left\{v^{n}\right\}_{1 / 4}+\operatorname{tr} \Gamma_{1} \boldsymbol{\nabla}\left\{u^{n}\right\}_{1 / 4}+\operatorname{tr} \Gamma_{3} \boldsymbol{\nabla}\left\{\psi^{n}\right\}_{1 / 4}+\operatorname{det} \Gamma_{1} \boldsymbol{\nabla}\left\{\Psi^{n}\right\}_{1 / 4} .
$$

By Lemma 3.1, $\left\{v^{n}\right\}_{1 / 4}=\left[u^{n}\right]_{\Delta t}$. Thus, with (2.16), the above yields:

$$
\begin{aligned}
\nabla\left\{q^{n}\right\}_{1 / 4} & =\nabla\left[u^{n}\right]_{\Delta t}+\left(\Gamma_{2}+2 \Gamma_{1}\right) \nabla\left\{u^{n}\right\}_{1 / 4}+\operatorname{tr} \Gamma_{3} \nabla\left\{\psi^{n}\right\}_{1 / 4}+\operatorname{det} \Gamma_{1} \nabla\left\{\Psi^{n}\right\}_{1 / 4} \\
& \stackrel{(3.9)}{=} \nabla\left[u^{n}\right]_{\Delta t}+\left[\phi^{n}\right]_{\Delta t}+\Gamma_{1}\left\{\phi^{n}\right\}_{1 / 4}-\Gamma_{3} \nabla\left\{\psi^{n}\right\}_{1 / 4}+2 \Gamma_{1} \boldsymbol{\nabla}\left\{u^{n}\right\}_{1 / 4} \\
& +\operatorname{tr} \Gamma_{3} \boldsymbol{\nabla}\left\{\psi^{n}\right\}_{1 / 4}+\operatorname{det} \Gamma_{1} \boldsymbol{\nabla}\left\{\Psi^{n}\right\}_{1 / 4} .
\end{aligned}
$$

Using (2.17) to substitute in the above $\operatorname{tr} \Gamma_{3} \mathrm{Id}-\Gamma_{3}$ and $\operatorname{det} \Gamma_{1}$, we obtain

$$
\begin{aligned}
\nabla\left\{q^{n}\right\}_{1 / 4} & =\left[\nabla u^{n}+\phi^{n}\right]_{\Delta t}+2 \Gamma_{1}\left\{\nabla u^{n}+\phi^{n}\right\}_{1 / 4}-\Gamma_{1}\left\{\phi^{n}\right\}_{1 / 4} \\
& +\Gamma_{1}\left(\Gamma_{1}+\Gamma_{2}\right) \nabla\left\{\psi^{n}\right\}_{1 / 4}+\Gamma_{1} \Gamma_{3} \nabla\left\{\Psi^{n}\right\}_{1 / 4} .
\end{aligned}
$$

With $\boldsymbol{\Lambda}^{n}$ defined in the statement of the lemma and the observations (3.8) and (3.15),

$$
\left[\boldsymbol{\Lambda}^{n}\right]_{\Delta t}=\boldsymbol{\nabla}\left[\psi^{n}\right]_{\Delta t}+\left[\boldsymbol{\Phi}^{n}\right]_{\Delta t}=\boldsymbol{\nabla}\left\{u^{n}\right\}_{1 / 4}+\left\{\boldsymbol{\phi}^{n}\right\}_{1 / 4} .
$$

Similarly, a direct computation, with the use of (3.7b) and (3.12) gives

$$
\left[\left[\boldsymbol{\Lambda}^{n}\right]\right]_{\Delta t}=\boldsymbol{\nabla}\left[u^{n}\right]_{\Delta t}+\left[\phi^{n}\right]_{\Delta t} .
$$

By expressing the first two terms in (3.22) via $\boldsymbol{\Lambda}^{n}$ and replacing $\left\{\boldsymbol{\phi}^{n}\right\}_{1 / 4}$ from (3.15), we obtain:

$$
\begin{aligned}
\boldsymbol{\nabla}\left\{q^{n}\right\}_{1 / 4} & =\left[\left[\boldsymbol{\Lambda}^{n}\right]\right]_{\Delta t}+2 \Gamma_{1}\left[\boldsymbol{\Lambda}^{n}\right]_{\Delta t}-\Gamma_{1}\left[\boldsymbol{\Phi}^{n}\right]_{\Delta t} \\
& +\Gamma_{1}\left(\Gamma_{1}+\Gamma_{2}\right) \boldsymbol{\nabla}\left\{\psi^{n}\right\}_{1 / 4}+\Gamma_{1} \Gamma_{3} \boldsymbol{\nabla}\left\{\Psi^{n}\right\}_{1 / 4} .
\end{aligned}
$$

Or, alternatively,

$$
\begin{aligned}
\boldsymbol{\nabla}\left\{q^{n}\right\}_{1 / 4}- & \left(\left[\left[\boldsymbol{\Lambda}^{n}\right]\right]_{\Delta t}+2 \Gamma_{1}\left[\boldsymbol{\Lambda}^{n}\right]_{\Delta t}+\Gamma_{1}^{2}\left\{\boldsymbol{\Lambda}^{n}\right\}_{1 / 4}\right)=\Gamma_{1}\left(-\Gamma_{1}\left\{\boldsymbol{\Phi}^{n}\right\}_{1 / 4}\right. \\
& \left.+\Gamma_{3} \boldsymbol{\nabla}\left\{\Psi^{n}\right\}_{1 / 4}+\Gamma_{2} \boldsymbol{\nabla}\left\{\psi^{n}\right\}_{1 / 4}-\left[\boldsymbol{\Phi}^{n}\right]_{\Delta t}\right) .
\end{aligned}
$$

The left-hand side of the above vanishes because of Lemma 3.5, which concludes the proof. 
Lemma 3.5 Let $\Psi^{n}, \boldsymbol{\Phi}^{n}$ be defined by (3.11), (3.12), with $\Psi^{0}, \boldsymbol{\Phi}^{0}$ satisfying (3.16). Then, $H^{n}=H^{0}$ for all $n \geq 0$, where

$$
H^{n}=\phi^{n}+\Gamma_{1} \Phi^{n}-\Gamma_{2} \nabla \psi^{n}-\Gamma_{3} \nabla \Psi^{n} .
$$

In particular, when $\xi_{i} \neq 0$ and $\{i, j, k\}=\{1,2,3\}$, we have

$$
\phi_{i}^{n}+\xi_{i} \Phi_{i}^{n}=\left(\xi_{j}+\xi_{k}-\xi_{i}\right) \partial_{x_{i}} \psi^{n}+\xi_{j} \xi_{k} \partial_{x_{i}} \Psi^{n},
$$

for all $n \geq 0$.

Proof Replacing the averages in (3.7c) by differences, using (3.12), (3.7b) and (3.11), yields

$$
\frac{\phi^{n+1}-\phi^{n}}{\Delta t}+\Gamma_{1} \frac{\boldsymbol{\Phi}^{n+1}-\boldsymbol{\Phi}^{n}}{\Delta t}=\Gamma_{2} \nabla \frac{\psi^{n+1}-\psi^{n}}{\Delta t}+\Gamma_{3} \nabla \frac{\Psi^{n+1}-\Psi^{n}}{\Delta t} .
$$

By multiplying (3.27) by $\Delta t$ and rearranging the terms we recover $H^{n+1}=H^{n}$. Since $n \geq 0$ is arbitrary, it follows that $H^{n}=H^{0}$, by induction. Owing to (3.16), we have $\Gamma_{1} H^{n}=\Gamma_{1} H^{0}=0$, and hence the conclusion.

Now we have all the ingredients necessary to prove Theorem 3.1.

Proof (Theorem 3.1) We proceed as in the proof of Theorem 2.2. From (3.17), we note that

$$
\begin{aligned}
{\left[q^{n}\right]_{\Delta t} } & =\left[v^{n}\right]_{\Delta t}+\operatorname{tr} \Gamma_{1}\left[u^{n}\right]_{\Delta t}+\operatorname{tr} \Gamma_{3}\left[\psi^{n}\right]_{\Delta t}+\operatorname{det} \Gamma_{1}\left[\Psi^{n}\right]_{\Delta t} \\
& =\left[\left[u^{n}\right]\right]_{\Delta t}+\operatorname{tr} \Gamma_{1}\left[u^{n}\right]_{\Delta t}+\operatorname{tr} \Gamma_{3}\left\{u^{n}\right\}_{1 / 4}+\operatorname{det} \Gamma_{1}\left\{\psi^{n}\right\}_{1 / 4},
\end{aligned}
$$

because of (3.13), (3.8), (3.14). Thus, (3.7a) reads

$$
\left[q^{n}\right]_{\Delta t}-\operatorname{div}\left(\nabla\left\{u^{n}\right\}_{1 / 4}+\left\{\phi^{n}\right\}_{1 / 4}\right)=0 .
$$

We test (3.7a) with $\left\{q^{n}\right\}_{1 / 4}$ and integrate by parts, noting that, similarly to the continuous case, either of the boundary conditions (3.10) yields no boundary terms (see discussion after (2.19)), and thus we get

$$
\frac{E_{k}^{n+1 / 2}-E_{k}^{n-1 / 2}}{\Delta t}+\left\langle\left\{\boldsymbol{\nabla} u^{n}+\phi^{n}\right\}_{1 / 4}, \nabla\left\{q^{n}\right\}_{1 / 4}\right\rangle=0,
$$

with $E_{k}^{\ell+1 / 2}$ given by (3.18). By Lemma 3.4 and (3.23), we have

$$
\left\langle\left\{\boldsymbol{\nabla} u^{n}+\phi^{n}\right\}_{1 / 4}, \boldsymbol{\nabla}\left\{q^{n}\right\}_{1 / 4}\right\rangle=\left\langle\left[\boldsymbol{\Lambda}^{n}\right]_{\Delta t},\left[\left[\boldsymbol{\Lambda}^{n}\right]\right]_{\Delta t}+2 \Gamma_{1}\left[\boldsymbol{\Lambda}^{n}\right]_{\Delta t}+\Gamma_{1}^{2}\left\{\boldsymbol{\Lambda}^{n}\right\}_{1 / 4}\right\rangle
$$

and thus by Lemma 3.2 we recover

$$
\begin{aligned}
\left\langle\left\{\boldsymbol{\nabla} u^{n}+\phi^{n}\right\}_{1 / 4}, \nabla\left\{q^{n}\right\}_{1 / 4}\right\rangle & =\frac{1}{2 \Delta t}\left(\left\|\frac{\boldsymbol{\Lambda}^{n+1}-\boldsymbol{\Lambda}^{n}}{\Delta t}\right\|^{2}+\left\|\Gamma_{1} \boldsymbol{\Lambda}^{n+\frac{1}{2}}\right\|^{2}\right. \\
& \left.-\left\|\frac{\boldsymbol{\Lambda}^{n}-\boldsymbol{\Lambda}^{n-1}}{\Delta t}\right\|^{2}-\left\|\Gamma_{1} \boldsymbol{\Lambda}^{n-\frac{1}{2}}\right\|^{2}\right)+2\left\|\left[\boldsymbol{\Lambda}^{n}\right]_{\Delta t}\right\|_{\Gamma_{1}}^{2} .
\end{aligned}
$$


From (3.7b) and (3.12), we recall that

$$
\frac{\Lambda^{n+1}-\Lambda^{n}}{\Delta t}=\nabla u^{n+\frac{1}{2}}+\phi^{n+\frac{1}{2}} .
$$

Thus, using (3.19) and (3.23), we obtain

$$
\left\langle\left\{\boldsymbol{\nabla} u^{n}+\phi^{n}\right\}_{1 / 4}, \nabla\left\{q^{n}\right\}_{1 / 4}\right\rangle=\frac{1}{\Delta t}\left(E_{p}^{n+\frac{1}{2}}-E_{p}^{n-\frac{1}{2}}\right)+2\left\|\left\{\boldsymbol{\nabla} u^{n}+\phi^{n}\right\}_{1 / 4}\right\|_{\Gamma_{1}}^{2} .
$$

Substitution of the above into the energy identity (3.29) concludes the proof.

This result implies that the discretization (3.7) is unconditionally stable; moreover, its energy mimics the energy of the continuous PML system (2.1).

\subsection{Explicit scheme}

In applications, explicit numerical methods are not only more convenient but also often more efficient than implicit schemes. To derive an explicit method, we first discretize (2.1) in space and then modify the previous implicit scheme (3.7).

\subsubsection{Spatial semi-discretization}

Starting from (2.1), we consider a Galerkin finite element (FE) discretization in space: the semi-discrete approximations of $u, \psi$ are denoted by $u_{h}, \psi_{h}$, and that of $\phi$ by $\phi_{h}$. Hence, we seek $u_{h}, \psi_{h}$ in $U_{h}=\operatorname{span}\left\{u_{j}, j=1, \ldots, n\right\} \subset H^{1}(\Omega)\left(\right.$ or $\left.H_{0}^{1}(\Omega)\right)$ and $\phi_{h} \in \boldsymbol{F}_{h} \subset\left(L^{2}(\Omega)\right)^{3}, \boldsymbol{F}_{h}=\operatorname{span}\left\{\boldsymbol{f}_{j}, j=1, \ldots, m\right\}$. We denote by $\langle\cdot, \cdot\rangle_{h}$ an (approximate) $L^{2}(\Omega)$ scalar product on $U_{h}$ defined using numerical quadratures, and for $\mathbf{v}_{h}, \mathbf{w}_{h} \in \boldsymbol{F}_{h}$, we denote

$$
\left\langle\mathbf{v}_{h}, \mathbf{w}_{h}\right\rangle_{h}:=\sum_{i=1}^{3}\left\langle\left(\mathbf{v}_{h}\right)_{i},\left(\mathbf{w}_{h}\right)_{i}\right\rangle_{h} .
$$

Next we introduce discrete operators on the finite-dimensional spaces $U_{h}$ and $\boldsymbol{F}_{h}$, defined by respective sesquilinear forms:

$$
\begin{array}{rlrl}
\nabla_{h}: U_{h} \rightarrow \boldsymbol{F}_{h}, & \left\langle\boldsymbol{\nabla}_{h} q_{h}, \mathbf{v}_{h}\right\rangle_{h}:=\left\langle\boldsymbol{\nabla} q_{h}, \mathbf{v}_{h}\right\rangle_{h}, & & \left(q_{h}, \mathbf{v}_{h}\right) \in U_{h} \times \boldsymbol{F}_{h}, \\
\operatorname{div}_{h}: \boldsymbol{F}_{h} \rightarrow U_{h}, \quad\left\langle\operatorname{div}_{h} \mathbf{v}_{h}, q_{h}\right\rangle_{h}:=-\left\langle\mathbf{v}_{h}, \boldsymbol{\nabla}_{h} q_{h}\right\rangle_{h}, & & \left(q_{h}, \mathbf{v}_{h}\right) \in U_{h} \times \boldsymbol{F}_{h}, \\
\Delta_{h}: U_{h} \rightarrow U_{h}, & \left\langle\Delta_{h} q_{h}, p_{h}\right\rangle_{h}:=-\left\langle\boldsymbol{\nabla}_{h} q_{h}, \boldsymbol{\nabla}_{h} p_{h}\right\rangle_{h}, & & \left(q_{h}, p_{h}\right) \in U_{h} \times U_{h} .
\end{array}
$$

Observe that by definition,

$$
\Delta_{h}=\operatorname{div}_{h} \nabla_{h}
$$

We emphasize that this property holds even if mass-lumping is used.

For the sake of simplicity we drop the subscript $h$ in what follows and denote by $\|\cdot\|$ the induced norm. The spatial semi-discretization of (2.1) for constant $\left\{\xi_{i}\right\}_{i=1}^{3}$ then reads:

$$
\left\{\begin{array}{l}
\partial_{t}^{2} u_{h}+\operatorname{tr} \Gamma_{1} \partial_{t} u_{h}+\operatorname{tr} \Gamma_{3} u_{h}+\operatorname{det} \Gamma_{1} \psi_{h}-\Delta_{h} u_{h}-\operatorname{div}_{h} \phi_{h}=0 \\
\partial_{t} \psi_{h}=u_{h} \\
\partial_{t} \phi_{h}+\Gamma_{1} \phi_{h}=\Gamma_{2} \nabla_{h} u_{h}+\Gamma_{3} \nabla_{h} \psi_{h}
\end{array}\right.
$$

Note that we need to replace the multiplications with $\operatorname{tr} \Gamma_{1}, \operatorname{tr} \Gamma_{2}, \operatorname{det} \Gamma_{1}, \Gamma_{1}, \Gamma_{2}$, $\Gamma_{3}$ by more complicated expressions when $\xi_{i} \neq$ const. 


\subsubsection{Explicit discretization and energy estimates}

To obtain a fully explicit scheme, we now replace in (3.7a) $\Delta\left\{u^{n}\right\}_{1 / 4}$ and $\operatorname{div}\left\{\phi^{n}\right\}_{1 / 4}$ by $\Delta u^{n}$ and $\operatorname{div} \phi^{n}$, respectively. Combined with the spatial semi-discretization (3.31), this results in the following fully discrete system:

$$
\begin{aligned}
& {\left[\left[u_{h}^{n}\right]\right]_{\Delta t}+\operatorname{tr} \Gamma_{1}\left[u_{h}^{n}\right]_{\Delta t}+\operatorname{tr} \Gamma_{3}\left\{u_{h}^{n}\right\}_{1 / 4}+\operatorname{det} \Gamma_{1}\left\{\psi_{h}^{n}\right\}_{1 / 4}} \\
& -\Delta_{h} u_{h}^{n}-\operatorname{div}_{h} \phi_{h}^{n}=0, \\
& \frac{\psi_{h}^{n+1}-\psi_{h}^{n}}{\Delta t}=\frac{u_{h}^{n+1}+u_{h}^{n}}{2}, \\
& \frac{\phi_{h}^{n+1}-\phi_{h}^{n}}{\Delta t}+\Gamma_{1} \frac{\phi_{h}^{n+1}+\phi_{h}^{n}}{2}=\Gamma_{2} \nabla_{h} \frac{u_{h}^{n+1}+u_{h}^{n}}{2}+\Gamma_{3} \nabla_{h} \frac{\psi_{h}^{n+1}+\psi_{h}^{n}}{2} .
\end{aligned}
$$

Remark 3.1 In contrast to the time discretization used in [26, 25], the zeroth order term in (3.32a) involving $\Gamma_{3}$ is not simply evaluated at the current time $t^{n}$ but instead replaced by the weighted time average $\left\{u_{h}^{n}\right\}_{1 / 4}$. This small distinction leads to a provably stable fully discrete numerical scheme for constant damping functions $\xi_{i}$. Numerical results with varying $\xi_{i}$ also suggest that the above formulation is more stable in the presence of steep gradients or high contrasts in the damping profiles.

To prove the stability of the above fully discrete explicit scheme under a certain CFL condition to be determined, we require the following algebraic identity.

Lemma 3.6 For any sequence $\left\{v^{n}\right\}_{n=0}^{\infty}$, it holds that

$$
v^{n}=\left\{v^{n}\right\}_{1 / 4}-\frac{\Delta t^{2}}{4}\left[\left[v^{n}\right]\right]_{\Delta t}, \quad n \geq 1
$$

We define $\boldsymbol{\Phi}_{h}, \Psi_{h}, v_{h}$ as in (3.11)-(3.13), with all the unknowns replaced by their discrete analogues, which therefore satisfy:

$$
\Psi_{h}^{0}=0, \quad \Gamma_{1}\left(\Gamma_{1} \Phi_{h}^{0}+\phi_{h}^{0}-\Gamma_{2} \nabla_{h} \psi_{h}^{0}\right)=0 .
$$

As in (3.17), (3.21), we let

$$
\begin{aligned}
& q_{h}^{n}=v_{h}^{n}+\operatorname{tr} \Gamma_{1} u_{h}^{n}+\operatorname{tr} \Gamma_{3} \psi_{h}^{n}+\operatorname{det} \Gamma_{1} \Psi_{h}^{n}, \\
& \boldsymbol{\Lambda}_{h}^{n}=\nabla_{h} \psi_{h}^{n}+\boldsymbol{\Phi}_{h}^{n}, \quad n \geq 0 .
\end{aligned}
$$

Due to the similarities between the semi-discrete implicit scheme of the previous section and the above explicit fully discrete scheme, some results obtained for the former also hold true for the latter. Thus to adapt these results to the explicit scheme, we need only add the subscript $h$ to the appropriate variables and operators. We refrain from repeating these results for the explicit scheme and instead simply refer to the previous results for the implicit scheme, with the understanding that the semi-discrete variables and spatial operators should be replaced by their appropriate discrete counterparts. 
With the above definitions, we introduce the following energy-related quantities - see also (3.18-3.20):

$$
\begin{aligned}
& E_{k, h}^{n+\frac{1}{2}}=\frac{1}{2}\left(\left\|q_{h}^{n+\frac{1}{2}}\right\|^{2}-\frac{\Delta t^{2}}{4}\left\|\nabla_{h} q_{h}^{n+\frac{1}{2}}\right\|^{2}\right), \\
& E_{p, h}^{n+\frac{1}{2}}=\frac{1}{2}\left(\left\|\nabla_{h} u_{h}^{n+\frac{1}{2}}+\phi_{h}^{n+\frac{1}{2}}\right\|^{2}+\left\|\Gamma_{1}\left(\nabla_{h} \psi_{h}^{n+\frac{1}{2}}+\boldsymbol{\Phi}_{h}^{n+\frac{1}{2}}\right)\right\|^{2}\right), \\
& E_{a d d, h}^{n+\frac{1}{2}}=\frac{(\Delta t)^{2}}{8}\left(\left\|\Gamma_{1} \frac{\boldsymbol{\Lambda}_{h}^{n+1}-\boldsymbol{\Lambda}_{h}^{n}}{\Delta t}\right\|^{2}+\left\|2 \Gamma_{1} \frac{\boldsymbol{\Lambda}_{h}^{n+1}-\boldsymbol{\Lambda}_{h}^{n}}{\Delta t}+\Gamma_{1}^{2} \boldsymbol{\Lambda}_{h}^{n+\frac{1}{2}}\right\|^{2}\right), \\
& E_{\text {expl }}^{n+\frac{1}{2}}=E_{k, h}^{n+\frac{1}{2}}+E_{p, h}^{n+\frac{1}{2}}+E_{a d d, h}^{n+\frac{1}{2}},
\end{aligned}
$$

where the subscript expl stands for 'explicit' whereas add stands for 'additional' to underline that this term does not appear in the expression for the energy of the implicit scheme. Note that $E_{\text {expl }}^{n+\frac{1}{2}}$ corresponds to a true (positive definite) energy provided that $E_{k, h}^{n+\frac{1}{2}} \geq 0$, that is under the (classical) CFL condition $C_{C F L} \leq 1$, where

$$
C_{C F L}:=\frac{\Delta t}{2} \max _{v_{h} \in U_{h}} \frac{\left\|\nabla_{h} v_{h}\right\|}{\left\|v_{h}\right\|} .
$$

With the above definitions, we can now formulate the following energy identity.

Theorem 3.2 For any solution $\left(u_{h}^{n}, \psi_{h}^{n}, \phi_{h}^{n}\right)$ of the initial value problem for (3.32), and $\boldsymbol{\Phi}_{h}^{n}, \Psi_{h}^{n}$ satisfying (3.33), it holds: for $n \geq 1$,

$$
\begin{aligned}
\frac{1}{\Delta t}\left(E_{\text {expl }}^{n+\frac{1}{2}}-E_{\text {expl }}^{n-\frac{1}{2}}\right) & =-\frac{\Delta t^{2}}{2}\left\|\left[\nabla_{h} u_{h}^{n}+\phi_{h}^{n}\right]_{\Delta t}\right\|_{\Gamma_{1}}^{2} \\
& -2\left\|\left\{\nabla_{h} u_{h}^{n}+\phi_{h}^{n}\right\}_{1 / 4}\right\|_{\Gamma_{1}}^{2} .
\end{aligned}
$$

For the proof, we need the following two auxiliary lemmas.

Lemma 3.7 For all $n \geq 1$,

$$
\left[\boldsymbol{\nabla}_{h} q_{h}^{n}\right]_{\Delta t}=\left[\left[\boldsymbol{\nabla}_{h} u_{h}^{n}+\boldsymbol{\phi}_{h}^{n}\right]\right]_{\Delta t}+2 \Gamma_{1}\left[\left[\boldsymbol{\Lambda}_{h}^{n}\right]\right]_{\Delta t}+\Gamma_{1}^{2}\left[\boldsymbol{\Lambda}_{h}^{n}\right]_{\Delta t} .
$$

Proof The proof follows the derivation of Lemma 3.4. First, we note that

$$
\left[\nabla_{h} q_{h}^{n}\right]_{\Delta t}=\left[\left[\nabla_{h} u_{h}^{n}\right]\right]_{\Delta t}+\operatorname{tr} \Gamma_{1}\left[\nabla_{h} u_{h}^{n}\right]_{\Delta t}+\operatorname{tr} \Gamma_{3}\left[\psi_{h}^{n}\right]_{\Delta t}+\operatorname{det} \Gamma_{1}\left[\nabla_{h} \Psi_{h}^{n}\right]_{\Delta t} .
$$

By using (2.16), we rewrite the above as

$$
\begin{aligned}
{\left[\nabla_{h} q_{h}^{n}\right]_{\Delta t} } & =\left[\left[\nabla_{h} u_{h}^{n}\right]\right]_{\Delta t}+\left(\Gamma_{2}+2 \Gamma_{1}\right)\left[\nabla_{h} u_{h}^{n}\right]_{\Delta t} \\
& +\operatorname{tr} \Gamma_{3}\left[\psi_{h}^{n}\right]_{\Delta t}+\operatorname{det} \Gamma_{1}\left[\nabla_{h} \Psi_{h}^{n}\right]_{\Delta t} .
\end{aligned}
$$

From (3.32c), we infer that

$$
\left[\left[\phi_{h}^{n}\right]\right]_{\Delta t}+\Gamma_{1}\left[\phi_{h}^{n}\right]_{\Delta t}-\Gamma_{3}\left[\nabla_{h} \psi_{h}^{n}\right]_{\Delta t}=\Gamma_{2}\left[\nabla_{h} u_{h}^{n}\right]_{\Delta t},
$$

which we use to replace $\Gamma_{2}\left[\nabla_{h} u_{h}^{n}\right]_{\Delta t}$ in (3.42). This yields

$$
\begin{aligned}
{\left[\nabla_{h} q_{h}^{n}\right]_{\Delta t} } & =\left[\left[\nabla_{h} u_{h}^{n}+\phi_{h}^{n}\right]\right]_{\Delta t}+\Gamma_{1}\left[\phi_{h}^{n}\right]_{\Delta t}-\Gamma_{3}\left[\nabla_{h} \psi_{h}^{n}\right]_{\Delta t} \\
& +2 \Gamma_{1}\left[\nabla_{h} u_{h}^{n}\right]_{\Delta t}+\operatorname{tr} \Gamma_{3}\left[\psi_{h}^{n}\right]_{\Delta t}+\operatorname{det} \Gamma_{1}\left[\nabla_{h} \Psi_{h}^{n}\right]_{\Delta t} .
\end{aligned}
$$


As in the proof of Lemma 3.4, we now substitute in the above $\operatorname{tr} \Gamma_{3} \operatorname{Id}-\Gamma_{3}$ and $\operatorname{det} \Gamma_{1}$ from (2.17):

$$
\begin{aligned}
{\left[\nabla_{h} q_{h}^{n}\right]_{\Delta t} } & =\left[\left[\nabla_{h} u_{h}^{n}+\phi_{h}^{n}\right]\right]_{\Delta t}+2 \Gamma_{1}\left[\nabla_{h} u_{h}^{n}+\phi_{h}^{n}\right]_{\Delta t}-\Gamma_{1}\left[\phi_{h}^{n}\right]_{\Delta t} \\
& +\Gamma_{1}\left(\Gamma_{1}+\Gamma_{2}\right)\left[\nabla_{h} \psi_{h}^{n}\right]_{\Delta t}+\Gamma_{1} \Gamma_{3}\left[\nabla_{h} \Psi_{h}^{n}\right]_{\Delta t} .
\end{aligned}
$$

Using (3.9) for discretized fields (which follows from (3.32c)), as well as (3.15),

$$
\left[\boldsymbol{\nabla}_{h} q_{h}^{n}\right]_{\Delta t}=\left[\left[\boldsymbol{\nabla}_{h} u_{h}^{n}+\boldsymbol{\phi}_{h}^{n}\right]\right]_{\Delta t}+2 \Gamma_{1}\left[\boldsymbol{\nabla}_{h} u_{h}^{n}+\boldsymbol{\phi}_{h}^{n}\right]_{\Delta t}+\Gamma_{1}^{2}\left\{\boldsymbol{\nabla}_{h} u_{h}^{n}+\boldsymbol{\phi}_{h}^{n}\right\}_{1 / 4} .
$$

The statement of the lemma follows from the above combined with (3.24) and (3.23) rewritten for the discrete case.

Lemma 3.8 For all $n \geq 1$, it holds

$$
\begin{aligned}
\left\langle\left[\left[\nabla_{h} u_{h}^{n}+\phi_{h}^{n}\right]\right]_{\Delta t},\left\{\nabla_{h} q_{h}^{n}\right\}_{1 / 4}\right\rangle & =\frac{1}{2 \Delta t}\left(\left\|\nabla_{h} q_{h}^{n+\frac{1}{2}}\right\|^{2}-\left\|\nabla_{h} q_{h}^{n-\frac{1}{2}}\right\|^{2}\right) \\
& -2\left\|\left[\nabla_{h} u_{h}^{n}+\phi_{h}^{n}\right]_{\Delta t}\right\|_{\Gamma_{1}}^{2}-\frac{4}{(\Delta t)^{3}}\left(E_{a d d, h}^{n+\frac{1}{2}}-E_{a d d, h}^{n-\frac{1}{2}}\right) .
\end{aligned}
$$

Proof From Lemma 3.7, we have

$$
\left[\left[\boldsymbol{\nabla}_{h} u_{h}^{n}+\boldsymbol{\phi}_{h}^{n}\right]\right]_{\Delta t}=\left[\boldsymbol{\nabla}_{h} q_{h}^{n}\right]_{\Delta t}-2 \Gamma_{1}\left[\left[\boldsymbol{\Lambda}_{h}^{n}\right]\right]_{\Delta t}-\Gamma_{1}^{2}\left[\boldsymbol{\Lambda}_{h}^{n}\right]_{\Delta t} .
$$

Using (3.4) we thus obtain

$$
\begin{aligned}
\left\langle\left[\left[\boldsymbol{\nabla}_{h} u_{h}^{n}+\phi_{h}^{n}\right]\right]_{\Delta t}, \nabla_{h}\left\{q^{n}\right\}_{1 / 4}\right\rangle & =\left\langle\left[\boldsymbol{\nabla}_{h} q_{h}^{n}\right]_{\Delta t}-2 \Gamma_{1}\left[\left[\boldsymbol{\Lambda}_{h}^{n}\right]\right]_{\Delta t}-\Gamma_{1}^{2}\left[\boldsymbol{\Lambda}_{h}^{n}\right]_{\Delta t},\left\{\boldsymbol{\nabla}_{h} q_{h}^{n}\right\}_{1 / 4}\right\rangle \\
& =\frac{1}{2 \Delta t}\left(\left\|\nabla_{h} q_{h}^{n+\frac{1}{2}}\right\|^{2}-\left\|\nabla_{h} q_{h}^{n-\frac{1}{2}}\right\|^{2}\right) \\
& -\left\langle 2 \Gamma_{1}\left[\left[\boldsymbol{\Lambda}_{h}^{n}\right]\right]_{\Delta t}+\Gamma_{1}^{2}\left[\boldsymbol{\Lambda}_{h}^{n}\right]_{\Delta t},\left\{\boldsymbol{\nabla}_{h} q_{h}^{n}\right\}_{1 / 4}\right\rangle .
\end{aligned}
$$

We now focus on the very last term in (3.43) and use Lemma 3.4 to express $\left\{\nabla_{h} q_{h}^{n}\right\}_{1 / 4}$ via $\boldsymbol{\Lambda}_{h}$ to obtain:

$$
\begin{gathered}
\left\langle 2 \Gamma_{1}\left[\left[\boldsymbol{\Lambda}_{h}^{n}\right]\right]_{\Delta t}+\Gamma_{1}^{2}\left[\boldsymbol{\Lambda}_{h}^{n}\right]_{\Delta t},\left\{\nabla_{h} q_{h}^{n}\right\}_{1 / 4}\right\rangle=\left\langle 2 \Gamma_{1}\left[\left[\boldsymbol{\Lambda}_{h}^{n}\right]\right]_{\Delta t}+\Gamma_{1}^{2}\left[\boldsymbol{\Lambda}_{h}^{n}\right]_{\Delta t},\left[\left[\boldsymbol{\Lambda}_{h}^{n}\right]\right]_{\Delta t}\right\rangle \\
+\left\langle 2 \Gamma_{1}\left[\left[\boldsymbol{\Lambda}_{h}^{n}\right]\right]_{\Delta t}+\Gamma_{1}^{2}\left[\boldsymbol{\Lambda}_{h}^{n}\right]_{\Delta t}, 2 \Gamma_{1}\left[\boldsymbol{\Lambda}_{h}^{n}\right]_{\Delta t}+\Gamma_{1}^{2}\left\{\boldsymbol{\Lambda}_{h}^{n}\right\}_{1 / 4}\right\rangle .
\end{gathered}
$$

From (3.5) it follows that

$$
\begin{aligned}
\left\langle 2 \Gamma_{1}\left[\left[\boldsymbol{\Lambda}_{h}^{n}\right]\right]_{\Delta t}\right. & \left.+\Gamma_{1}^{2}\left[\boldsymbol{\Lambda}_{h}^{n}\right]_{\Delta t},\left\{\boldsymbol{\nabla}_{h} q_{h}^{n}\right\}_{1 / 4}\right\rangle=2 \Gamma_{1}\left\|\left[\left[\boldsymbol{\Lambda}_{h}^{n}\right]\right]_{\Delta t}\right\|^{2} \\
& +\frac{1}{2 \Delta t}\left(\left\|\Gamma_{1} \frac{\boldsymbol{\Lambda}_{h}^{n+1}-\boldsymbol{\Lambda}_{h}^{n}}{\Delta t}\right\|^{2}-\left\|\Gamma_{1} \frac{\boldsymbol{\Lambda}_{h}^{n}-\boldsymbol{\Lambda}_{h}^{n-1}}{\Delta t}\right\|^{2}\right) \\
& +\left\langle 2 \Gamma_{1}\left[\left[\boldsymbol{\Lambda}_{h}^{n}\right]\right]_{\Delta t}+\Gamma_{1}^{2}\left[\boldsymbol{\Lambda}_{h}^{n}\right]_{\Delta t}, 2 \Gamma_{1}\left[\boldsymbol{\Lambda}_{h}^{n}\right]_{\Delta t}+\Gamma_{1}^{2}\left\{\boldsymbol{\Lambda}_{h}^{n}\right\}_{1 / 4}\right\rangle .
\end{aligned}
$$

Finally, with (3.6), we obtain

$$
\begin{aligned}
\left\langle 2 \Gamma_{1}\left[\left[\boldsymbol{\Lambda}_{h}^{n}\right]\right]_{\Delta t}\right. & \left.+\Gamma_{1}^{2}\left[\boldsymbol{\Lambda}_{h}^{n}\right]_{\Delta t},\left\{\nabla_{h} q_{h}^{n}\right\}_{1 / 4}\right\rangle=2\left\|\left[\left[\boldsymbol{\Lambda}_{h}^{n}\right]\right]_{\Delta t}\right\|_{\Gamma_{1}}^{2} \\
& +\frac{1}{2 \Delta t}\left(\left\|\Gamma_{1} \frac{\boldsymbol{\Lambda}_{h}^{n+1}-\boldsymbol{\Lambda}_{h}^{n}}{\Delta t}\right\|^{2}+\left\|2 \Gamma_{1} \frac{\boldsymbol{\Lambda}_{h}^{n+1}-\boldsymbol{\Lambda}_{h}^{n}}{\Delta t}+\Gamma_{1}^{2} \frac{\boldsymbol{\Lambda}_{h}^{n+1}+\boldsymbol{\Lambda}_{h}^{n}}{2}\right\|^{2}\right. \\
& \left.-\left\|\Gamma_{1} \frac{\boldsymbol{\Lambda}_{h}^{n}-\boldsymbol{\Lambda}_{h}^{n-1}}{\Delta t}\right\|^{2}-\left\|2 \Gamma_{1} \frac{\boldsymbol{\Lambda}_{h}^{n}-\boldsymbol{\Lambda}_{h}^{n-1}}{\Delta t}+\Gamma_{1}^{2} \frac{\boldsymbol{\Lambda}_{h}^{n}+\boldsymbol{\Lambda}_{h}^{n-1}}{2}\right\|^{2}\right) .
\end{aligned}
$$


To conclude the proof, we combine the above expression with (3.43) and recall that $\left[\left[\boldsymbol{\Lambda}_{h}^{n}\right]\right]_{\Delta t}=\left[\boldsymbol{\nabla}_{h} u_{h}^{n}+\boldsymbol{\phi}_{h}^{n}\right]_{\Delta t}(\operatorname{see}(3.24))$.

Now we can prove the principal result of this section, namely Theorem 3.2.

Proof (Theorem 3.2) By proceeding as in the proof of Theorem 3.1 and using (3.30), we obtain the following identity:

$$
\frac{\left\|q_{h}^{n+\frac{1}{2}}\right\|^{2}-\left\|q_{h}^{n-\frac{1}{2}}\right\|^{2}}{2 \Delta t}+\left\langle\nabla_{h} u_{h}^{n}+\phi_{h}^{n}, \nabla_{h}\left\{q_{h}^{n}\right\}_{1 / 4}\right\rangle=0 .
$$

Let us remark that to obtain the above, we use the fact that due to either of boundary conditions (3.10), integration by parts yields no boundary terms (see discussion after $(2.19)$ ). On applying Lemma 3.6 to $\nabla_{h} u_{h}^{n}+\phi_{h}^{n}$, the above transforms into

$$
\begin{aligned}
& \frac{\left\|q_{h}^{n+\frac{1}{2}}\right\|^{2}-\left\|q_{h}^{n-\frac{1}{2}}\right\|^{2}}{2 \Delta t}+\left\langle\left\{\nabla_{h} u_{h}^{n}+\phi_{h}^{n}\right\}_{1 / 4}, \nabla_{h}\left\{q_{h}^{n}\right\}_{1 / 4}\right\rangle \\
& -\frac{\Delta t^{2}}{4}\left\langle\left[\left[\nabla_{h} u_{h}^{n}+\phi_{h}^{n}\right]\right]_{\Delta t}, \nabla_{h}\left\{q_{h}^{n}\right\}_{1 / 4}\right\rangle=0 .
\end{aligned}
$$

By proceeding as in the proof of Theorem 3.1 and using (3.36) to express the first two terms in the above via $E_{k, h}^{n \pm \frac{1}{2}}$, we rewrite (3.44) as follows:

$$
\begin{aligned}
& \frac{1}{\Delta t}\left(E_{k, h}^{n+\frac{1}{2}}-E_{k, h}^{n-\frac{1}{2}}\right)+\frac{\Delta t}{8}\left(\left\|\nabla_{h} q_{h}^{n+\frac{1}{2}}\right\|^{2}-\left\|\nabla_{h} q_{h}^{n-\frac{1}{2}}\right\|^{2}\right) \\
& +\frac{1}{\Delta t}\left(E_{p, h}^{n+\frac{1}{2}}-E_{p, h}^{n-\frac{1}{2}}\right)+2\left\|\left\{\nabla_{h} u_{h}^{n}+\phi_{h}^{n}\right\}_{1 / 4}\right\|_{\Gamma_{1}}^{2} \\
& -\frac{\Delta t^{2}}{4}\left\langle\left[\left[\nabla_{h} u_{h}^{n}+\phi_{h}^{n}\right]\right]_{\Delta t}, \nabla_{h}\left\{q_{h}^{n}\right\}_{1 / 4}\right\rangle=0 .
\end{aligned}
$$

Substitution of the last term using Lemma 3.8 finally yields (3.41).

\subsection{Control of unknowns}

Here, we demonstrate that the norms of the unknown discrete fields do not grow in time when the explicit time discretization (3.32) is used, which corresponds to the discrete counterpart of Theorem 2.3.

Theorem 3.3 Let $\left(u_{h}^{n}, \phi_{h}^{n}, \psi_{h}^{n}\right)$ solve the initial-value problem for (3.32) with $\xi_{1}, \xi_{2}$, $\xi_{3} \geq 0$ and $C_{C F L}<1$, where the $C F L$ constant $C_{C F L}$ is given by (3.40). Then there exists a constant $C>0$, which depends only on the initial data, the damping functions $\xi_{j}, j=1,2,3$ and $C_{C F L}$, such that

- if $\xi_{i} \neq 0$ for some $i \in\{1,2,3\}$, then

$$
\left\|u_{h}^{n}\right\|+\left\|\nabla_{h} u_{h}^{n+\frac{1}{2}}\right\|+\left\|\frac{u_{h}^{n+1}-u_{h}^{n}}{\Delta t}\right\|+\left\|\phi_{h}^{n}\right\| \leq C, \quad \text { for all } n \geq 0 .
$$

- if, additionally, $\xi_{j} \neq 0$ for some $j \neq i \in\{1,2,3\}$, then

$$
\left\|\psi_{h}^{n}\right\|+\left\|\nabla_{h} \psi_{h}^{n}\right\| \leq C, \quad \text { for all } n \geq 0 .
$$


Again the proof relies on several auxiliary lemmas. The following result mimics Lemma 2.3.

Lemma 3.9 Let $v_{h}^{n} \in U_{h}, n \geq 0$, and let the sequence $w^{n+\frac{1}{2}}$ be defined by

$$
w^{n+\frac{1}{2}}=\frac{v^{n+1}-v^{n}}{\Delta t}+\gamma \frac{v^{n+1}+v^{n}}{2}, \quad \gamma>0, \Delta t>0 .
$$

If there exists a constant $C_{w}>0$, s.t. $\left\|w^{k+1 / 2}\right\| \leq C_{w}$ uniformly for all $k \geq 0$, then the following bounds hold uniformly for all $n \geq 0$ :

$$
\left\|v^{n}\right\| \leq\left\|v^{0}\right\|+C_{v}, \quad\left\|\frac{v^{n+1}-v^{n}}{\Delta t}\right\| \leq \gamma\left\|v^{0}\right\|+C_{v}^{\prime} .
$$

Here the constants $C_{v}, C_{v}^{\prime}>0$ only depend on $C_{w}$ and $\gamma$.

Proof From (3.45), we have

$$
v^{n+1}=\left(\frac{1}{\Delta t}+\frac{\gamma}{2}\right)^{-1} w^{n+\frac{1}{2}}+v^{n} \nu, \quad \nu=\left(1-\frac{\gamma \Delta t}{2}\right)\left(1+\frac{\gamma \Delta t}{2}\right)^{-1} .
$$

Hence,

$$
v^{n+1}=v^{0} \nu^{n+1}+\sum_{\ell=0}^{n} w^{n-\ell+1 / 2} \nu^{\ell}\left(\frac{1}{\Delta t}+\frac{\gamma}{2}\right)^{-1} .
$$

Since $|\nu|<1$, the above implies the uniform bound (3.46) for $\left\|v^{n}\right\|$. By applying the triangle inequality to (3.45) and using the uniform bound for $w^{n+\frac{1}{2}}$, we get:

$$
\left\|\frac{v^{n+1}-v^{n}}{\Delta t}\right\| \leq C_{w}+\gamma\left\|\frac{v^{n+1}+v^{n}}{2}\right\|,
$$

which, together with $\left\|v^{n}\right\| \leq\left\|v^{0}\right\|+C_{v}$, results in the second bound in (3.46). Note that all constants are also uniformly bounded in $\Delta t$.

Next, we need the discrete counterpart of Lemma 2.4.

Lemma 3.10 Let $\left(u_{h}^{n}, \psi_{h}^{n}, \phi_{h}^{n}\right)$ solve the initial-value problem for (3.32) with $\xi_{1}, \xi_{2}$, $\xi_{3} \geq 0$, and $C_{C F L}<1$ with $C_{C F L}$ given by (3.40). Then there exists a constant $C>0$, which depends only on the initial data and $\xi_{j}, j=1,2,3$, such that

- if $\xi_{i} \neq 0$, then for all $n \geq 0$,

$$
\begin{aligned}
& \left\|u_{h}^{n}\right\| \leq C, \quad(3.47 \mathrm{a}) \\
& \text { additionally, } \xi_{j} \neq 0, \text { for } j \neq i, \text { then }
\end{aligned}\left\|\frac{u_{h}^{n+1}-u_{h}^{n}}{\Delta t}\right\| \leq C .
$$

- if, additionally, $\xi_{j} \neq 0$, for $j \neq i$, then

$$
\left\|\psi_{h}^{n}\right\| \leq C, \quad n \geq 0 .
$$

Proof Again, we let $C$ denote a generic constant that depends on the initial data, damping functions and the CFL only. Theorem 3.2, combined with the assumption $C_{C F L}<1$, implies the following uniform bound in $\ell$ :

$$
\left\|q_{h}^{\ell+\frac{1}{2}}\right\| \leq C, \quad \ell \geq 0 .
$$

Next, we consider the following three separate cases: 
$-\xi_{i} \neq 0$ and $\xi_{j}=\xi_{k}=0, i \neq j \neq k$. The bounds (3.47a, 3.47b) follow from

$$
q_{h}^{n+\frac{1}{2}}=v_{h}^{n+\frac{1}{2}}+\xi_{i} u_{h}^{n+\frac{1}{2}}=\frac{u_{h}^{n+1}-u_{h}^{n}}{\Delta t}+\xi_{i} u_{h}^{n+\frac{1}{2}}
$$

(see (3.13) for the definition of $v_{h}^{n}$ ) and Lemma 3.9.

$-\xi_{i}, \xi_{j} \neq 0$ and $\xi_{k}=0$. Here we need to show (3.47a), (3.47b) and (3.48) following similar ideas as previously. First, we recall that

$$
q_{h}^{n+\frac{1}{2}}=\frac{u_{h}^{n+1}-u_{h}^{n}}{\Delta t}+\left(\xi_{i}+\xi_{j}\right) u_{h}^{n+\frac{1}{2}}+\xi_{i} \xi_{j} \psi_{h}^{n+\frac{1}{2}} .
$$

Next, we define the auxiliary unknown,

$$
g_{h}^{n}=u_{h}^{n}+\xi_{i} \psi_{h}^{n},
$$

which yields

$$
g_{h}^{n+\frac{1}{2}}=u_{h}^{n+\frac{1}{2}}+\xi_{i} \psi_{h}^{n+\frac{1}{2}}=\frac{\psi_{h}^{n+1}-\psi_{h}^{n}}{\Delta t}+\xi_{i} \psi_{h}^{n+\frac{1}{2}}
$$

where the last identity follows from (3.32b). We also have

$$
\frac{g_{h}^{n+1}-g_{h}^{n}}{\Delta t}=\frac{u_{h}^{n+1}-u_{h}^{n}}{\Delta t}+\xi_{i} u_{h}^{n+\frac{1}{2}} .
$$

Therefore, we can rewrite (3.49) as

$$
q_{h}^{n+\frac{1}{2}}=\frac{g_{h}^{n+1}-g_{h}^{n}}{\Delta t}+\xi_{j} g_{h}^{n+\frac{1}{2}} .
$$

By applying Lemma 3.9, we deduce that for all $n \geq 0$,

$$
\left\|g_{h}^{n}\right\| \leq C, \quad(3.53) \quad\left\|\frac{g_{h}^{n+1}-g_{h}^{n}}{\Delta t}\right\| \leq C .
$$

With (3.53), Lemma 3.9 applied to (3.51) yields the bound (3.48). The uniform bound (3.47a) follows from the triangle inequality applied to (3.50), and the bound (3.47b) follows from (3.54) and the triangle inequality applied to (3.52) using (3.47a).

$-\xi_{1}, \xi_{2}, \xi_{3} \neq 0$. We will only sketch the proof, since it is very similar to the previous case, and consists in multiple applications of Lemma 2.3. Let us first define an auxiliary unknown $w_{h}^{n}$ :

$$
w_{h}^{n}=u_{h}^{n}+\left(\xi_{2}+\xi_{1}\right) \psi_{h}^{n}+\xi_{1} \xi_{2} \Psi_{h}^{n} .
$$

Then, with (3.32b) and (3.11), we have

$$
\begin{aligned}
\frac{w_{h}^{n+1}-w_{h}^{n}}{\Delta t}+\xi_{3} w_{h}^{n+\frac{1}{2}} & =\frac{u_{h}^{n+1}-u_{h}^{n}}{\Delta t}+\left(\xi_{2}+\xi_{1}\right) u_{h}^{n+\frac{1}{2}}+\xi_{1} \xi_{2} \psi_{h}^{n+\frac{1}{2}} \\
& +\xi_{3} u_{h}^{n+\frac{1}{2}}+\xi_{3}\left(\xi_{2}+\xi_{1}\right) \psi_{h}^{n+\frac{1}{2}}+\xi_{1} \xi_{2} \xi_{3} \Psi_{h}^{n+\frac{1}{2}}
\end{aligned}
$$

Upon comparison with (3.34), we obtain

$$
q_{h}^{n+\frac{1}{2}}=\frac{w_{h}^{n+1}-w_{h}^{n}}{\Delta t}+\xi_{3} w_{h}^{n+\frac{1}{2}} .
$$


Next, we let

$$
G_{h}^{n}=\psi_{h}^{n}+\xi_{2} \Psi_{h}^{n}
$$

and verify that

$$
\frac{G_{h}^{n+1}-G_{h}^{n}}{\Delta t}+\xi_{1} G_{h}^{n+\frac{1}{2}}=w_{h}^{n+\frac{1}{2}},
$$

and

$$
\frac{G_{h}^{n+1}-G_{h}^{n}}{\Delta t}=\frac{\psi_{h}^{n+1}-\psi_{h}^{n}}{\Delta t}+\xi_{2} \psi_{h}^{n+\frac{1}{2}},
$$

Then the desired result follows by multiple applications of Lemma 2.3, first to (3.57) (to bound $\left\|w_{h}^{n}\right\|$ and $\left\|\frac{w_{h}^{n+1}-w_{h}^{n}}{\Delta t}\right\|$ ), next to (3.59) (to bound $\left\|G_{h}^{n}\right\|$ and $\left\|\frac{G_{h}^{n+1}-G_{h}^{n}}{\Delta t}\right\|$ ), and finally to (3.60) (which permits to bound $\left\|\psi_{h}^{n}\right\|$, thus obtaining (3.48)). Applying the triangle inequality to (3.58), we obtain a uniform bound on $\left\|\Psi_{h}^{n}\right\|$; next, the triangle inequality with (3.55) gives us the uniform bound (3.47a) on $\left\|u_{h}^{n}\right\|$. Finally, to get (3.47b), it suffices to apply the triangle inequality to $(3.56)$.

The following lemma shows that we also control the discrete norms of the derivatives. For conciseness, we shall henceforth use the following notation:

$$
\nabla_{h} u_{h}=\left(\partial_{x_{1}}^{h} u_{h}, \partial_{x_{2}}^{h} u_{h}, \partial_{x_{3}}^{h} u_{h}\right) \text {. }
$$

Lemma 3.11 Let $\left(u_{h}^{n}, \phi_{h}^{n}, \psi_{h}^{n}\right)$ solve the initial-value problem for (3.32) with $\xi_{1}, \xi_{2}$, $\xi_{3} \geq 0$ and $C_{C F L}<1$ with $C_{C F L}$ as in (3.40). Then there exists a constant $C>0$, which depends only on the initial data, $\xi_{j}, j=1,2,3$, and $C_{C F L}$, such that

- we have

$$
\left\|\nabla_{h} u_{h}^{n+\frac{1}{2}}\right\| \leq C, \quad \text { for all } n \geq 0
$$

- if, additionally, for some $i \in\{1,2,3\}, \xi_{i} \neq 0$, then

$$
\left\|\phi_{h}^{n}\right\| \leq C, \quad n \geq 0 .
$$

- if, additionally, $\xi_{j} \neq 0$, for some $j \neq i$, then

$$
\left\|\nabla_{h} \psi_{h}^{n}\right\| \leq C, \quad n \geq 0 .
$$

Proof Again, we let $C>0$ denote a generic constant that depends only on the initial data, $C_{C F L}$ and $\xi_{j}, j=1,2,3$. Due to the assumptions of the lemma together with Theorem 3.2, the following uniform bounds hold:

$$
\begin{aligned}
& \left\|\Gamma_{1}\left(\nabla_{h} \psi_{h}^{n+\frac{1}{2}}+\boldsymbol{\Phi}_{h}^{n+\frac{1}{2}}\right)\right\| \leq C, \\
& \left\|\nabla_{h} u_{h}^{n+\frac{1}{2}}+\phi_{h}^{n+\frac{1}{2}}\right\| \leq C, \quad n \geq 0 .
\end{aligned}
$$

Let us consider the following four separate cases. 
$-\xi_{1}=\xi_{2}=\xi_{3}=0$. Then (3.61) is a direct consequence of Theorem 3.2, see Remark 2.2.

$-\xi_{i} \neq 0$, and $\xi_{j}=\xi_{k}=0, i \neq j \neq k, i, j, k \in\{1,2,3\}$. Without loss of generality, let us assume $i=1$. We can split the proof into two cases:

1. Uniform bounds for $\left\|\partial_{x_{1}}^{h} u_{h}^{n+\frac{1}{2}}\right\|$ and $\left\|\phi_{h, 1}^{n}\right\|$. Let us consider (3.26) in its fully discrete form written for $i=1$; it clearly yields

$$
\phi_{h, 1}^{n+\frac{1}{2}}+\xi_{1} \Phi_{h, 1}^{n+\frac{1}{2}}=-\xi_{1} \partial_{x_{1}}^{h} \psi_{h}^{n+\frac{1}{2}} .
$$

From the above expression and (3.64), we deduce that $\left\|\phi_{h, 1}^{n+\frac{1}{2}}\right\|$ is bounded uniformly in $n$, which together with (3.65) implies the bound on $\partial_{x_{1}}^{h} u_{h}^{n+\frac{1}{2}}$. To bound $\left\|\phi_{h, 1}^{n}\right\|$, we use (3.32c) written for $\phi_{h, 1}$, namely,

$$
\frac{\phi_{h, 1}^{n+1}-\phi_{h, 1}^{n}}{\Delta t}+\xi_{1} \phi_{h, 1}^{n+\frac{1}{2}}=-\xi_{1} \partial_{x_{1}}^{h} u_{h}^{n+\frac{1}{2}},
$$

and apply to it Lemma 3.9, as the right hand side is bounded uniformly in $n$.

2. Uniform bounds for $\left\|\partial_{x_{\ell}}^{h} u_{h}^{n+\frac{1}{2}}\right\|$ and $\left\|\phi_{h, \ell}^{n}\right\|, \ell \neq 1$. First, note that (3.32c) written for $\phi_{h, 2}$ reads:

$$
\frac{\phi_{h, 2}^{n+1}-\phi_{h, 2}^{n}}{\Delta t}=\xi_{1} \partial_{x_{2}}^{h} u_{h}^{n+\frac{1}{2}}
$$

Adding to both sides of the above expression $\xi_{1} \phi_{h, 2}^{n+\frac{1}{2}}$, we obtain

$$
\frac{\phi_{h, 2}^{n+1}-\phi_{h, 2}^{n}}{\Delta t}+\xi_{1} \phi_{h, 2}^{n+\frac{1}{2}}=\xi_{1} \partial_{x_{2}}^{h} u_{h}^{n+\frac{1}{2}}+\xi_{1} \phi_{h, 2}^{n+\frac{1}{2}}
$$

The right-hand side of this equation is bounded uniformly in $n$ due to (3.65). With Lemma 3.9, we obtain the uniform bound (3.62) on $\left\|\phi_{h, 2}^{n}\right\|$, as well as the uniform bound on $\left\|\frac{\phi_{h, 2}^{n+1}-\phi_{h, 2}^{n}}{\Delta t}\right\|$. The latter, combined with (3.66), immediately implies the uniform bound (3.61) for $\left\|\partial_{x_{2}}^{h} u_{h}^{n+\frac{1}{2}}\right\|$.

$-\xi_{i} \xi_{j} \neq 0, \xi_{k} \equiv 0$, for $i \neq j \neq k$. In this case we will demonstrate (3.61), (3.62) and (3.63). Without loss of generality, let $i=1, j=2$ and $k=3$.

1. Uniform bounds for $\left\|\partial_{x_{\ell}}^{h} u_{h}^{n+\frac{1}{2}}\right\|,\left\|\phi_{h, \ell}^{n}\right\|,\left\|\partial_{x_{\ell}}^{h} \psi_{h}^{n}\right\|, \ell=1,2$. Without loss of generality, we show the bounds for $\ell=1$, as the proofs are essentially identical for $\ell=2$.

From (3.26) with $i=1$, we have

$$
\phi_{h, 1}^{n+\frac{1}{2}}+\xi_{1} \Phi_{h, 1}^{n+\frac{1}{2}}=\left(\xi_{2}-\xi_{1}\right) \partial_{x_{1}}^{h} \psi_{h}^{n+\frac{1}{2}} .
$$

Adding to both sides of the above $\partial_{x_{1}}^{h} u_{h}^{n+\frac{1}{2}}$ results in

$$
\phi_{h, 1}^{n+\frac{1}{2}}+\partial_{x_{1}}^{h} u^{n+\frac{1}{2}}+\xi_{1} \Phi_{h, 1}^{n+\frac{1}{2}}+\xi_{1} \partial_{x_{1}}^{h} \psi_{h}^{n+\frac{1}{2}}=\xi_{2} \partial_{x_{1}}^{h} \psi_{h}^{n+\frac{1}{2}}+\partial_{x_{1}}^{h} u_{h}^{n+\frac{1}{2}}
$$


By using (3.32b), we can rewrite the above as

$$
\phi_{h, 1}^{n+\frac{1}{2}}+\partial_{x_{1}}^{h} u^{n+\frac{1}{2}}+\xi_{1} \Phi_{h, 1}^{n+\frac{1}{2}}+\xi_{1} \partial_{x_{1}}^{h} \psi_{h}^{n+\frac{1}{2}}=\frac{\partial_{x_{1}}^{h} \psi_{h}^{n+1}-\partial_{x_{1}}^{h} \psi_{h}^{n}}{\Delta t}+\xi_{2} \partial_{x_{1}}^{h} \psi_{h}^{n+\frac{1}{2}} .
$$

By (3.64) and (3.65), the left-hand side is bounded uniformly in $n$. The application of Lemma 3.9 then implies for all $n \geq 0$ that

$$
\left\|\partial_{x_{1}}^{h} \psi_{h}^{n}\right\| \leq C, \quad\left\|\partial_{x_{1}}^{h} \frac{\psi_{h}^{n+1}-\psi_{h}^{n}}{\Delta t}\right\| \equiv\left\|\partial_{x_{1}}^{h} u_{h}^{n+\frac{1}{2}}\right\| \leq C .
$$

Finally, to get a uniform bound on $\left\|\phi_{h, 1}^{n}\right\|$, it suffices to apply Lemma 3.9 to (3.32c) written for $\phi_{h, 1}$ :

$$
\frac{\phi_{h, 1}^{n+1}-\phi_{h, 1}^{n}}{\Delta t}+\xi_{1} \phi_{h, 1}^{n+\frac{1}{2}}=\left(\xi_{2}-\xi_{1}\right) \partial_{x_{1}}^{h} u_{h}^{n+\frac{1}{2}} .
$$

2. Uniform bounds for $\left\|\partial_{x_{3}}^{h} u_{h}^{n+\frac{1}{2}}\right\|,\left\|\phi_{h, 3}^{n}\right\|$ and $\left\|\partial_{x_{3}}^{h} \psi_{h}^{n}\right\|$. Since $\xi_{3}=0$, we may not use (3.26) here, though we can use the first part of Lemma 3.5, which yields the following identity:

$$
\phi_{h, 3}^{n+1}-\phi_{h, 3}^{0}=\left(\xi_{1}+\xi_{2}\right) \partial_{x_{3}}^{h} \psi_{h}^{n+1}-\left(\xi_{1}+\xi_{2}\right) \partial_{x_{3}}^{h} \psi_{h}^{0}+\xi_{1} \xi_{2} \partial_{x_{3}}^{h} \Psi_{h}^{n+1},
$$

which, in its turn, yields

$$
\phi_{h, 3}^{n+\frac{1}{2}}-\phi_{h, 3}^{0}+\left(\xi_{1}+\xi_{2}\right) \partial_{x_{3}}^{h} \psi_{h}^{0}=\left(\xi_{1}+\xi_{2}\right) \partial_{x_{3}}^{h} \psi_{h}^{n+\frac{1}{2}}+\xi_{1} \xi_{2} \partial_{x_{3}}^{h} \Psi_{h}^{n+\frac{1}{2}} .
$$

Next, we add to both sides of the above $\partial_{x_{3}}^{h} u_{h}^{n+\frac{1}{2}}$ to obtain

$$
\begin{aligned}
\phi_{h, 3}^{n+\frac{1}{2}}+\partial_{x_{3}}^{h} u_{h}^{n+\frac{1}{2}} & -\phi_{h, 3}^{0}+\left(\xi_{1}+\xi_{2}\right) \partial_{x_{3}}^{h} \psi_{h}^{0}=\partial_{x_{3}}^{h} u_{h}^{n+\frac{1}{2}} \\
& +\left(\xi_{1}+\xi_{2}\right) \partial_{x_{3}}^{h} \psi_{h}^{n+\frac{1}{2}}+\xi_{1} \xi_{2} \partial_{x_{3}}^{h} \Psi_{h}^{n+\frac{1}{2}}
\end{aligned}
$$

The left hand side of the above is bounded because of (3.65). As for the right-hand side, one can easily verify that it can be rewritten as follows:

$$
\partial_{x_{3}}^{h} u_{h}^{n+\frac{1}{2}}+\left(\xi_{1}+\xi_{2}\right) \partial_{x_{3}}^{h} \psi_{h}^{n+\frac{1}{2}}+\xi_{1} \xi_{2} \partial_{x_{3}}^{h} \Psi_{h}^{n+\frac{1}{2}}=\frac{G_{h}^{n+1}-G_{h}^{n}}{\Delta t}+\xi_{1} G_{h}^{n+\frac{1}{2}},
$$

where

$$
G_{h}^{n}=\partial_{x_{3}} \psi_{h}^{n}+\xi_{2} \partial_{x_{3}} \Psi_{h}^{n},
$$

as in the proof of Lemma 3.10, case $\xi_{i}, \xi_{j} \neq 0$ and $\xi_{k}=0$, for instance.

By applying Lemma 3.9 to (3.69), we now deduce that the following two bounds hold uniformly in $n$ :

$$
\left\|G_{h}^{n}\right\| \leq C, \quad\left\|\frac{G_{h}^{n+1}-G_{h}^{n}}{\Delta t}\right\| \leq C .
$$


Again by applying Lemma 3.9 to (3.70) rewritten as

we deduce that

$$
G_{h}^{n+1 / 2}=\partial_{x_{3}} \frac{\Psi_{h}^{n+1}-\Psi_{h}^{n}}{\Delta t}+\xi_{2} \partial_{x_{3}} \Psi_{h}^{n+1 / 2},
$$

$$
\left\|\partial_{x_{3}} \Psi_{h}^{n}\right\| \leq C, \text { for all } n \geq 0 .
$$

Next, Lemma 3.9 applied to

yields the following bounds, for some constant $C>0$ :

$$
\frac{G_{h}^{n+1}-G_{h}^{n}}{\Delta t}=\partial_{x_{3}} \frac{\psi_{h}^{n+1}-\psi_{h}^{n}}{\Delta t}+\xi_{2} \partial_{x_{3}} \psi_{h}^{n+\frac{1}{2}},
$$

$$
\left\|\partial_{x_{3}} \psi_{h}^{n}\right\| \leq C, \quad\left\|\partial_{x_{3}} \frac{\psi_{h}^{n+1}-\psi_{h}^{n}}{\Delta t}\right\| \equiv\left\|\partial_{x_{3}} u_{h}^{n+\frac{1}{2}}\right\| \leq C, \text { for all } n \geq 0 .
$$

Finally, to get the bound on $\left\|\phi_{h, 3}^{n}\right\|$, we use the triangle inequality in (3.67), combined with the above uniform bound on $\left\|\partial_{x_{3}} \psi_{h}^{n}\right\|$ and (3.71).

- Finally, it remains to consider the case $\xi_{1}, \xi_{2}, \xi_{3}>0$. Let us first obtain the bounds on $\left\|\partial_{x_{1}}^{h} u_{h}^{n+\frac{1}{2}}\right\|,\left\|\partial_{x_{1}}^{h} \psi_{h}^{n}\right\|$ and $\left\|\phi_{h, 1}^{n}\right\|$, as the bounds for the remaining terms are similar. Here, we shall only sketch the proof, since it is very similar to previously used arguments.

From (3.26) for $i=1$, we have

$$
\phi_{h, 1}^{n+\frac{1}{2}}+\xi_{1} \Phi_{h, 1}^{n+\frac{1}{2}}=\left(\xi_{2}+\xi_{3}-\xi_{1}\right) \partial_{x_{1}}^{h} \psi_{h}^{n+\frac{1}{2}}+\xi_{2} \xi_{3} \partial_{x_{1}}^{h} \Psi_{h}^{n+\frac{1}{2}},
$$

or, after adding to both sides of the above $\partial_{x_{1}}^{h} u_{h}^{n+\frac{1}{2}}+\xi_{1} \partial_{x_{1}}^{h} \psi_{h}^{n+\frac{1}{2}}$,

$$
\begin{aligned}
\left(\phi_{h, 1}^{n+\frac{1}{2}}+\partial_{x_{1}}^{h} u_{h}^{n+\frac{1}{2}}\right)+ & \xi_{1}\left(\Phi_{h, 1}^{n+\frac{1}{2}}+\partial_{x_{1}}^{h} \psi_{h}^{n+\frac{1}{2}}\right)=\partial_{x_{1}}^{h} u_{h}^{n+\frac{1}{2}} \\
& +\left(\xi_{2}+\xi_{3}\right) \partial_{x_{1}}^{h} \psi_{h}^{n+\frac{1}{2}}+\xi_{2} \xi_{3} \partial_{x_{1}}^{h} \Psi_{h}^{n+\frac{1}{2}} .
\end{aligned}
$$

With

$$
G_{h}^{n}=\partial_{x_{1}}^{h} \psi_{h}^{n}+\xi_{3} \partial_{x_{1}}^{h} \Psi_{h}^{n},
$$

it is not difficult to verify (see the proof of Lemma 3.10 , case $\xi_{i}, \xi_{j} \neq 0$ and $\left.\xi_{k}=0\right)$ that

$$
\left(\phi_{h, 1}^{n+\frac{1}{2}}+\partial_{x_{1}}^{h} u_{h}^{n+\frac{1}{2}}\right)+\xi_{1}\left(\Phi_{h, 1}^{n+\frac{1}{2}}+\partial_{x_{1}}^{h} \psi_{h}^{n+\frac{1}{2}}\right)=\frac{G_{h}^{n+1}-G_{h}^{n}}{\Delta t}+\xi_{2} G_{h}^{n+\frac{1}{2}}
$$

Thanks to (3.64), (3.65) and Lemma 3.9, we obtain

$$
\left\|G_{h}^{n}\right\| \leq C, \quad\left\|\frac{G_{h}^{n+1}-G_{h}^{n}}{\Delta t}\right\| \leq C, \quad \text { for all } n \geq 0 .
$$

Hence, the desired bounds for $\left\|\partial_{x_{1}}^{h} u_{h}^{n+\frac{1}{2}}\right\|$ and $\left\|\partial_{x_{1}}^{h} \psi_{h}^{n}\right\|$ can be obtained similarly to those in (3.72). Finally, to get a uniform bound on $\left\|\phi_{h, 1}^{n}\right\|$, we apply Lemma 3.9 to the left-hand side of $(3.32 \mathrm{c})$ written for $\phi_{h, 1}^{n}$.

Proof (Theorem 3.3) The proof is a direct corollary of Lemmas 3.10 and 3.11. 


\section{Numerical results}

Here we perform a series of numerical experiments where we compute the solution of the 3D PML system (2.1) in the unit cube $\Omega=[0,1]^{3}$ using the explicit scheme described in Section 3.2. First, we consider damping functions $\xi_{i}$ that are constant throughout $\Omega$ to validate the theory. Next, we consider the realistic situation of piecewise constant damping functions that identically vanish inside the region of interest, $\Omega_{0}$.

For the spatial discretization, we use standard sixth-order hexahedral $\mathbb{Q}_{6}$-finite elements, which leads to approximately $1.4 \cdot 10^{7}$ degrees of freedom. The time step is set to $\Delta t \approx 0.001$, which corresponds to approximately $95 \%$ of the allowed maximal time step. We set the initial conditions to zero and consider either Neumann or Dirichlet conditions at the outer boundary $B$ of the PML for the sake of completeness.

To initiate an outward propagating spherical wave, we include in (2.1) the essentially compactly supported Gaussian source centered about $x_{0}$,

$$
\begin{aligned}
& f(t, \mathbf{x})=f_{0} \mathrm{e}^{-\sigma_{x}\left\|\mathbf{x}-\mathbf{x}_{0}\right\|^{2}} \frac{d}{d t}\left(\mathrm{e}^{-\sigma_{t}\left(t-t_{0}\right)^{2}}\right), \\
& \sigma_{x}=2 \cdot 10^{3}, \sigma_{t}=5 \cdot 10^{4}, \mathbf{x}_{0}=\left(\frac{1}{2}, \frac{1}{2}, \frac{1}{2}\right), t_{0}=0.02, f_{0}=10^{2} .
\end{aligned}
$$

The source $f(t, \mathbf{x})$ only acts during the very short time interval [0,0.04] while its amplitude lies below machine precision past $t=0.08$. In the simulations below, all quantities of interest are therefore computed after those first 80 time steps, that is for $t_{n}>0.08$ when $f$ is essentially zero and our theory is valid. The discrete energy $E_{\text {expl }}^{n+\frac{1}{2}}$ defined in (3.39), in particular, then satisfies the identity in Theorem 3.2 for $n \geq 80$.

\subsection{Constant damping coefficients}

We consider the situation of constant damping functions, where $\xi_{1}, \xi_{2}$ and $\xi_{3}$ are constant throughout $\Omega$; hence, the PML occupies the entire computational domain. We perform two sets of experiments:

- PML in a single direction, either with Dirichlet or Neumann conditions, with $\xi_{1}=40, \xi_{2}=\xi_{3}=0$.

- PML in all three directions, corresponding to a corner situation, either with Dirichlet or Neumann conditions, with $\xi_{1}=40, \xi_{2}=45, \xi_{3}=50$.

In Fig. 4.1, we observe that the decay rate of the energy is only algebraic for the PML in a single direction, while for the PML in all three directions (corner) the energy decays exponentially fast. To further validate Theorem 3.2, we evaluate the relative error

$$
\begin{aligned}
\epsilon^{n}=\left(\frac{1}{\Delta t}\right. & \left(E_{\text {expl }}^{n+\frac{1}{2}}-E_{\text {expl }}^{n-\frac{1}{2}}\right) \\
& \left.\quad+\frac{(\Delta t)^{2}}{2}\left\|\left[\nabla_{h} u_{h}^{n}+\phi_{h}^{n}\right]_{\Delta t}\right\|_{\Gamma_{1}}^{2}+2\left\|\left\{\nabla u_{h}^{n}+\phi_{h}^{n}\right\}_{1 / 4}\right\|_{\Gamma_{1}}^{2}\right) / E_{\text {expl }}^{n+\frac{1}{2}} .
\end{aligned}
$$



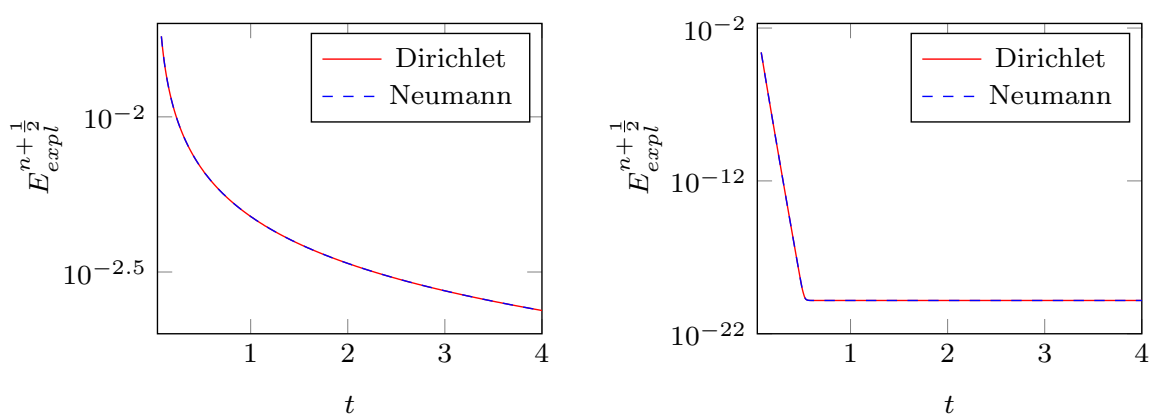

Fig. 4.1 Constant damping functions. Left: the energy $E_{\text {expl }}^{n+\frac{1}{2}}$ defined in (3.39) for the PML in a single direction, computed either with Dirichlet or Neumann conditions. Right: the energy $E_{\text {expl }}^{n+\frac{1}{2}}$ for the PML in all three directions, computed either with Dirichlet or Neumann boundary conditions.

In all our computations, $\epsilon^{n}$ never exceeded $10^{-12}$ thereby demonstrating the validity of Theorem 3.2 down to machine precision.

\subsection{Variable damping coefficients}

We consider the realistic situation of varying damping functions, when our theory is in fact not valid. More precisely, we choose $\xi_{i}$ piecewise-constant as

$$
\xi_{i}\left(x_{i}\right)=\left\{\begin{array}{l}
40, x_{i} \leq 0.1 \text { or } x_{i}>0.9, \quad i=1,2,3 . \\
0 \quad \text { otherwise, }
\end{array}\right.
$$

Hence $\Omega_{0}=[0.1,0.9]^{3}$ and the PML has width 0.1 in each direction, while the FE mesh is aligned with the boundary $B$ of the perfectly matched layer to avoid spurious reflections due to the discretization. In Fig. 4.2, snapshots of the numerical solution with a Dirichlet boundary condition are shown at different times. We recall that at $t=0.2$, the source is essentially zero. The spherical wave front enters the PML around $t \approx 0.4$ and is fully absorbed by time $t=0.8$ without any noticeable reflections. In contrast to similar experiments performed elsewhere, we did not observe any instabilities or spurious reflections when using discontinuous damping profiles. Indeed, as shown in the left frame of Fig. 4.3, we observe no spurious oscillations as the solution enters the PML. At later times, however, we observe in the right frame of Fig. 4.3 a thousandfold smaller back propagating reflection caused by the outer boundary of the PML. The absence of any spurious oscillations at the interface is probably due to the use of a very high 6 -th order FE discretization.

In the left frame of Fig. 4.4, we display the time evolution of the discrete energy $E_{\text {expl }}^{n+\frac{1}{2}}$ for piecewise constant damping functions that identically vanish inside $\Omega_{0}$, using either Dirichlet or Neumann boundary conditions. In the right frame, we show the discrete rate of change of the energy,

$$
\delta_{h}^{n}=\frac{E_{\text {expl }}^{n+\frac{1}{2}}-E_{\text {expl }}^{n-\frac{1}{2}}}{\Delta t} .
$$



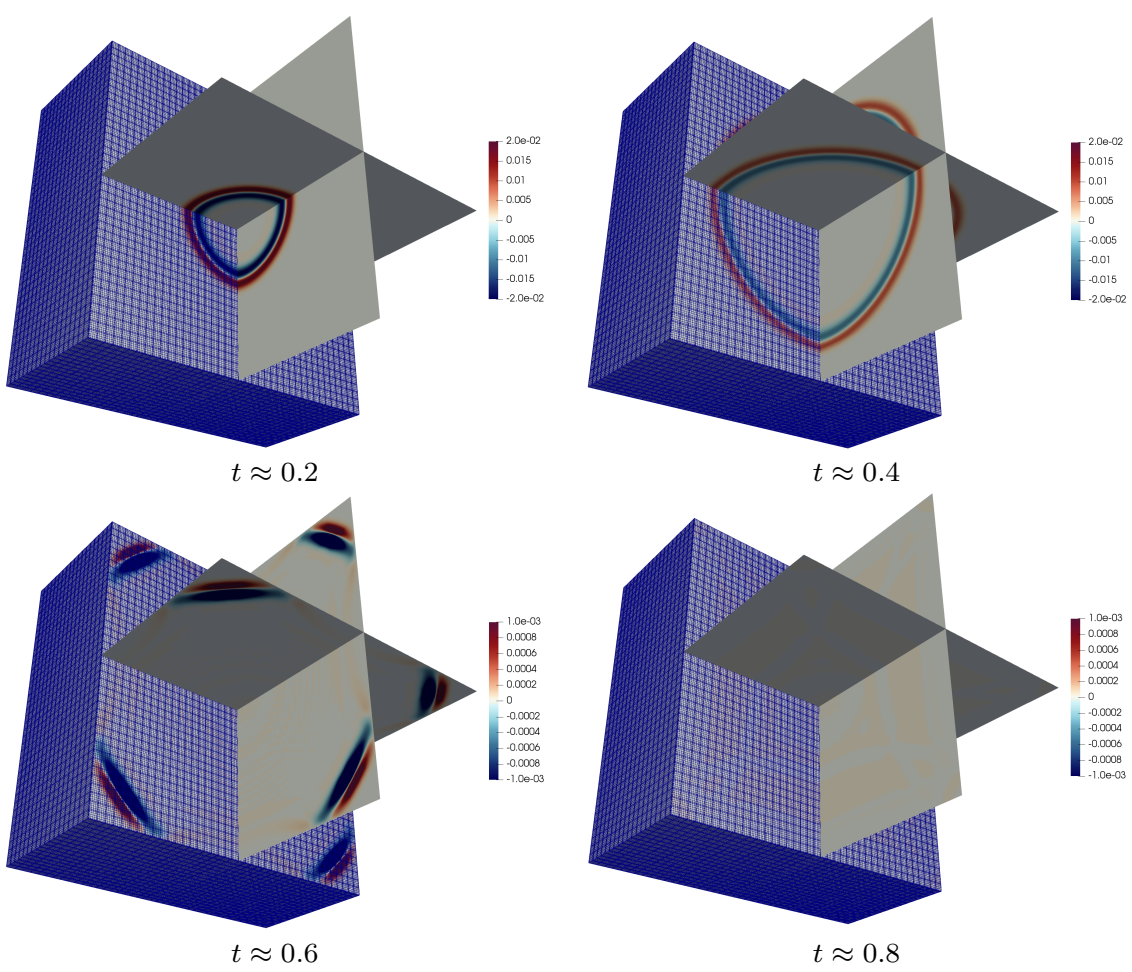

Fig. 4.2 Piecewise constant damping functions. Snapshots of the numerical solution at times $t \simeq 0.2,0.4,0.6,0.8$.
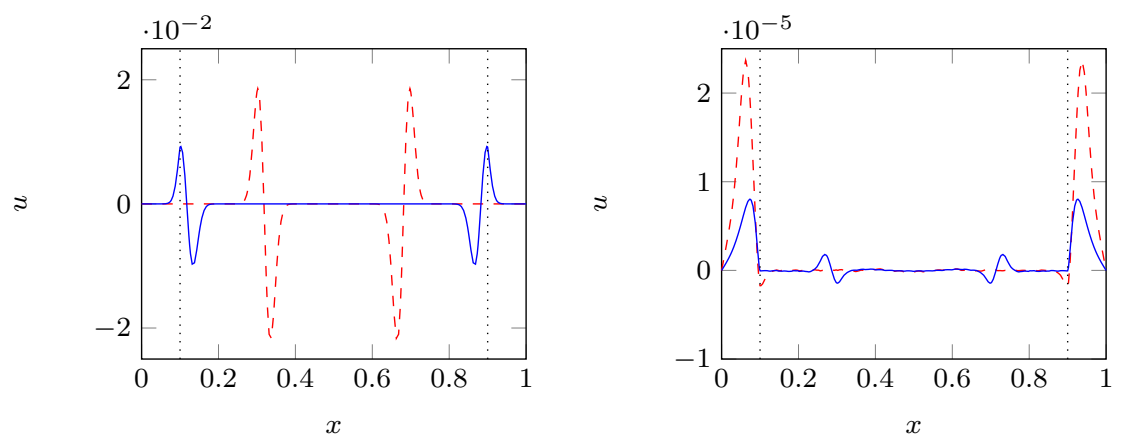

$$
---t \approx 0.2-t \approx 0.4
$$

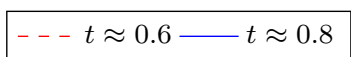

Fig. 4.3 Piecewise constant damping functions. Snapshots of $u\left(t, x, \frac{1}{2}, \frac{1}{2}\right)$ for $x \in(0,1)$. Left: $t \simeq 0.2,0.4$, right: $t \simeq 0.6,0.8$. Notice the difference in figure scales.

Clearly, we no longer expect $\epsilon_{n}$ defined in (4.2) to vanish identically. Still, we wish to investigate whether the energy $E_{\text {expl }}^{n+\frac{1}{2}}$ defined in (3.39) nonetheless decays in a situation of varying damping functions, that is whether $\delta_{h}^{n}$ in (4.3) remains negative. 

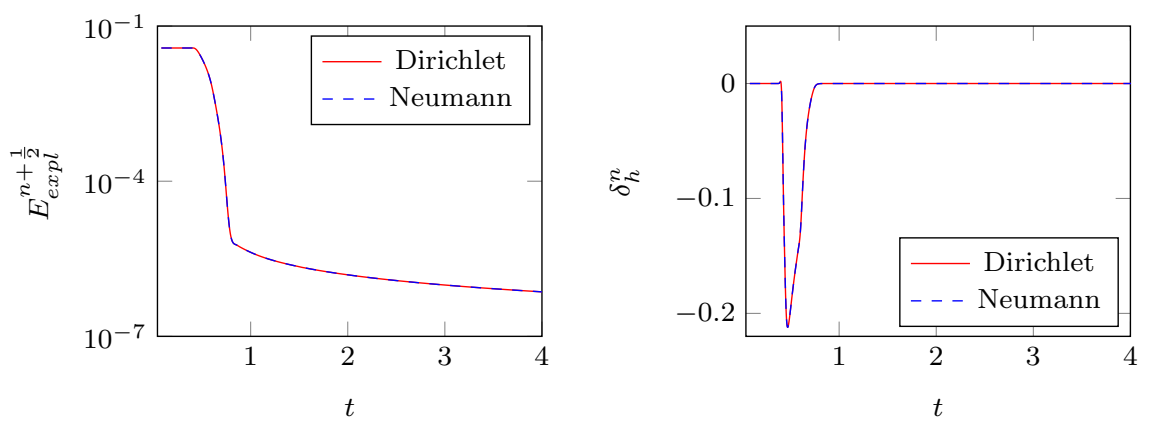

Fig. 4.4 Piecewise constant damping functions, short time. Left: the energy $E_{\text {expl }}^{n+\frac{1}{2}}$, defined in (3.39), computed either with Dirichlet or Neumann conditions. Right: the discrete rate of change in the energy $\delta_{h}^{n}$, defined in (4.3), computed either with Dirichlet or Neumann conditions.

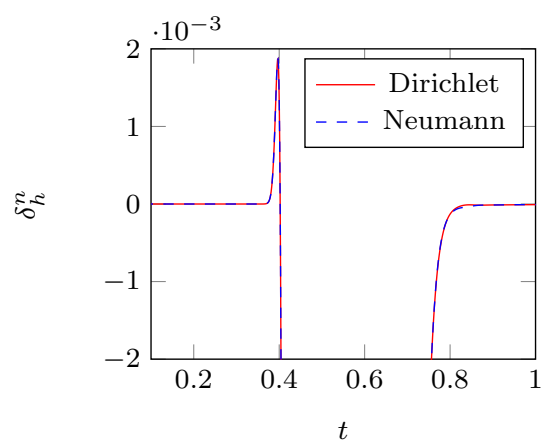

Fig. 4.5 Piecewise constant damping functions, a closer look. The discrete rate of change of the energy $\delta_{h}^{n}$ computed either with Dirichlet or Neumann conditions. Zoom on the right frame of Fig. 4.4 .

In Fig. 4.4, it appears at first that the energy still decays even in a situation of varying damping profiles. However, as we take a closer look in Fig. 4.5 at the time evolution of the discrete rate of change of the energy, we observe that in fact the energy no longer monotonically decreases. Indeed at time $t \approx 0.4$, that is when the wave front first penetrates the PML, $\delta_{h}^{n}$ exhibits an albeit small but positive maximum, though it remains strictly negative at all later times.

Finally, we demonstrate the long-time stability of our perfectly matched layer by performing a much longer simulation until time $t=36$. All parameters remain identical, except that we choose a FE mesh twice as coarse with about $1.8 \cdot 10^{6}$ degrees of freedom and a time-step about twice as large, $\Delta t \approx 0.002$. In Fig. 4.6, we observe that the energy (3.39) remains bounded and essentially decays during the entire simulation, be it with Dirichlet or Neumann conditions. Note that the energy essentially vanishes beyond time $t=0.8$, once the spherical wave has left the physical domain $\Omega_{0}$. 


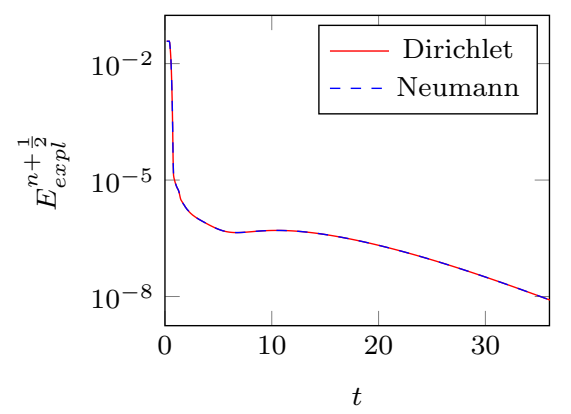

Fig. 4.6 Piecewise constant damping functions, long time. The discrete energy (3.39) computed either with Dirichlet or Neumann conditions.

\section{Concluding remarks}

Starting from the PML formulation from $[26,25]$ for the wave equation in its standard second-order form, we have proved energy decay first in two and then in three space dimensions for a judicious space-time energy functional. Our energy estimates apply in the full 3D setting including corners and imply boundedness of all the unknowns in the $L^{2}$-norm. Although we assume constant damping functions inside the PML for our analysis, our estimates pave the way for establishing stability in more general situations with variable damping functions or nonlinear dispersive terms.

We have also proposed a fully explicit discrete formulation which is provably stable for constant damping functions. The time-stepping scheme is based on the well-known leapfrog method and is stable under a CFL stability condition which is independent of the damping parameters inside the PML. The present time discretization slightly differs from that used in $[26,25]$ and appears more stable in numerical computations - see Remark 3.1.

Our numerical results for constant damping coefficients validate the theory to machine precision. The energy decays algebraically in the PML single layer formulation and exponentially in the PML corner formulation. We have also tested numerically the scheme for PMLs with piecewise constant damping functions. The implementation of the present formulation for this case is rather straightforward since it does not require transmission conditions to be treated explicitly. Although the conclusions of the theory are no longer valid for piecewise constant damping functions, our numerical results show that the energy still essentially decays even for very long times. They also illustrate that smooth or even just continuous damping functions are not necessary to achieve perfect matching at the discrete level.

Acknowledgements The fourth author acknowledges the partial support of a public grant as part of the Investissement d'avenir project, reference ANR-11-LABX-0056-LMH, LabEx LMH. 


\section{References}

1. Abarbanel, S., Gottlieb, D.: A mathematical analysis of the PML method. J. Comp. Phys. 134, 357-363 (1997)

2. Abarbanel, S., Gottlieb, D., Hestahaven, J.S.: Well-posed perfectly matched layers for advective acoustics. J. Comput. Phys. 154, 266-283 (1999)

3. Appelö, D., Hagstrom, T., Kreiss, G.: Perfectly matched layers for hyperbolic systems: general formulation, well-posedness, and stability. SIAM J. Appl. Math. 67(1), 1-23 (2006)

4. Appelö, D., Kreiss, G.: Application of a perfectly matched layer to the nonlinear wave equation. Wave Motion 44, 531-548 (2007)

5. Barucq, H., Diaz, J., Tlemcani, M.: New absorbing layers conditions for short water waves. J. Comput. Phys. 229(1), 58-72 (2010). DOI 10.1016/j.jcp.2009.08.033. URL https: //doi.org/10.1016/j.jcp.2009.08.033

6. Bécache, E., Fauqueux, S., Joly, P.: Stability of perfectly matched layers, group velocities and anisotropic waves. J. Comp. Phys. 188, 399-433 (2003)

7. Bécache, E., Joly, P.: On the analysis of Bérenger's perfectly matched layers for Maxwell's equations. M2AN Math. Model. Numer. Anal. 36(1), 87-119 (2002)

8. Bécache, E., Joly, P., Kachanovska, M.: Stable perfectly matched layers for a cold plasma in a strong background magnetic field. J. Comput. Phys. 341, 76-101 (2017). DOI 10.1016/j.jcp.2017.03.051. URL https://doi.org/10.1016/j.jcp.2017.03.051

9. Bécache, É., Joly, P., Kachanovska, M., Vinoles, V.: Perfectly matched layers in negative index metamaterials and plasmas. In: CANUM 2014-42e Congrès National d'Analyse Numérique, ESAIM Proc. Surveys, vol. 50, pp. 113-132. EDP Sci., Les Ulis (2015)

10. Bécache, E., Joly, P., Vinoles, V.: On the analysis of perfectly matched layers for a class of dispersive media and application to negative index metamaterials. Mathematics of Computations 87(314), 2775-2810 (2018)

11. Bécache, E., Kachanovska, M.: Stable perfectly matched layers for a class of anisotropic dispersive models. Part I: Necessary and sufficient conditions of stability. ESAIM Math. Model. Numer. Anal. 51(6), 2399-2434 (2017)

12. Bécache, E., Petropoulos, P.G., Gedney, S.D.: On the long-time behavior of unsplit perfectly matched layers. IEEE Trans. Antennas and Propagation 52(5), 1335-1342 (2004)

13. Bérenger, J.P.: A perfectly matched layer for the absorption of electromagnetic waves. J. Comput. Phys. 114(2), 185-200 (1994)

14. Bérenger, J.P.: Three-dimensional perfectly matched layer for the absorption of electromagnetic waves. J. Comput. Phys. 127(2), 363-379 (1996)

15. Chabassier, J., Imperiale, S.: Space/time convergence analysis of a class of conservative schemes for linear wave equations. C. R. Math. Acad. Sci. Paris 355(3), 282-289 (2017). DOI 10.1016/j.crma.2016.12.009. URL https://doi.org/10.1016/j.crma.2016.12.009

16. Chen, Z.: Convergence of the time-domain perfectly matched layer method for acoustic scattering problems. Int. J. Numer. Anal. Model. 6(1), 124-146 (2009)

17. Chew, W.C., Weedon, W.H.: A 3D perfectly matched medium from modified Maxwell's equations with stretched coordinates. Microwave and Optical Technology Letters 7(13), 599-604 (1994)

18. Collino, F., Monk, P.B.: Optimizing the perfectly matched layer. Comput. Methods Appl. Mech. Engrg. 164(1-2), 157-171 (1998). DOI 10.1016/S0045-7825(98)00052-8. URL https://doi.org/10.1016/S0045-7825(98)00052-8. Exterior problems of wave propagation (Boulder, CO, 1997; San Francisco, CA, 1997)

19. Collino, F., Tsogka, C.: Application of the perfectly matched absorbing layer model to the linear elastodynamic problem in anisotropic heterogeneous media. Geophysics 66, 294-307 (2001)

20. Demaldent, E., Imperiale, S.: Perfectly matched transmission problem with absorbing layers: application to anisotropic acoustics in convex polygonal domains. Internat. J. Numer. Methods Engrg. 96(11), 689-711 (2013). DOI 10.1002/nme.4572. URL https: //doi.org/10.1002/nme.4572

21. Diaz, J., Joly, P.: A time domain analysis of PML models in acoustics. Comput. Methods Appl. Mech. Engrg. 195(29-32), 3820-3853 (2006)

22. Duru, K.: The role of numerical boundary procedures in the stability of perfectly matched layers. SIAM J. Sci. Comput. 38(2), A1171-A1194 (2016). DOI 10.1137/140976443. URL https://doi.org/10.1137/140976443

23. Duru, K., Kreiss, G.: Boundary waves and stability of the perfectly matched layer for the two space dimensional elastic wave equation in second order form. SIAM Numer. Anal. 52, 2883-2904 (2014) 
24. Ervedoza, S., Zuazua, E.: Perfectly matched layers in 1-d: energy decay for continuous and semi-discrete waves. Numer. Math. 109(4), 597-634 (2008)

25. Grote, M., Sim, I.: Efficient PML for the wave equation. preprint, arXiv:1001.0319 [math:NA] (2010)

26. Grote, M.J., Sim, I.: Perfectly matched layer for the second-order wave equation. In: Proceedings of the Ninth International Conference on Numerical Aspects of Wave Propagation (WAVES 2009, held in Pau, France, 2009), pp. 370-371

27. Hagstrom, T., Appelö, D.: Automatic symmetrization and energy estimates using local operators for partial differential equations. Comm. Partial Differential Equations 32(7-9), 1129-1145 (2007)

28. Hu, F.Q.: On absorbing boundary conditions for linearized Euler equations by a perfectly matched layer. J. Comput. Phys. 129, 201-219 (1996)

29. Hu, F.Q.: A stable, perfectly matched layer for linearized Euler equations in unsplit physical variables. J. Comput. Phys. 173(2), 455-480 (2001). DOI 10.1006/jcph.2001.6887. URL https://doi.org/10.1006/jcph.2001.6887

30. Joly, P.: An elementary introduction to the construction and the analysis of perfectly matched layers for time domain wave propagation. SeMA J. (57), 5-48 (2012)

31. Kachanovska, M.: Stable perfectly matched layers for a class of anisotropic dispersive models. Part II: Energy estimates (2017). URL https://hal.inria.fr/hal-01419682. Https://hal.inria.fr/hal-01419682

32. Kaltenbacher, B., Kaltenbacher, M., Sim, I.: A modified and stable version of a perfectly matched layer technique for the 3-d second order wave equation in time domain with an application to aeroacoustics. J. Comput. Phys. 235, 407-422 (2013). DOI 10.1016/j.jcp. 2012.10.016. URL https://doi.org/10.1016/j.jcp.2012.10.016

33. Komatitsch, D., Tromp, J.: A perfectly matched layer absorbing boundary conditionfor the second-order seismic wave equation. Geophysics J. Int. 154, 146-153 (2003)

34. Nataf, F.: A new approach to perfectly matched layers for the linearized Euler system J. Comput. Phys. 214(2), 757-772 (2006). DOI 10.1016/j.jcp.2005.10.014. URL https : //doi.org/10.1016/j.jcp.2005.10.014

35. Sjögreen, B., Petersson, N.A.: Perfectly matched layers for Maxwell's equations in second order formulation. J. Comp. Phys. 209, 19-46 (2005)

36. Zhao, L., Cangellaris, A.C.: Gt-pml: generalized theory of perfectly matched layers and its application to the reflectionless truncation of finite-difference time-domain grids. IEEE Transactions on Microwave Theory and Techniques 44(12), 2555-2563 (1996) 